\title{
Fluidization of 1204 spheres: simulation and experiment
}

\author{
T.-W. Pan ${ }^{1}$, D.D. Joseph ${ }^{3}$, R. Bai ${ }^{3}$, R. Glowinski ${ }^{1}$, V. Sarin ${ }^{2}$ \\ ${ }^{1}$ Department of Mathematics, University of Houston, Houston, TX 77204 \\ ${ }^{2}$ Department of Computer Sciences, Texas A\&M University, College Station, TX 77843 \\ ${ }^{3}$ Department of Aerospace Engineering and Mechanics, University of Minnesota, Minneapolis, MN 55454
}

April 2000, Revised June 2001

\begin{abstract}
In this paper we study the fluidization of 1204 spheres at Reynolds numbers in the thousands using the method of distributed Lagrange multipliers. The results of the simulation are compared with a real experiment. This is the first direct numerical simulation of a real fluidized bed at the finite Reynolds number encountered in the applications. The simulations are processed like real experiments for straight lines in lot-log plots leading to power laws as in celebrated correlations of Richardson and Zaki [1954]. The numerical method allows for the first ever direct calculation of the slip velocity and other averaged values used in two-fluid continuum models. The computation and the experiment show that a single particle may be in balance under weight and drag for an interval of fluidizing velocities; the expectation that the fluidizing velocity is unique is not realized. The numerical method reveals that the dynamic pressure actually decreases slowly with the fluidizing velocity. Tentative interpretations of these new results are discussed.
\end{abstract}

\section{Introduction}

The current popularity of computational fluid dynamics is rooted in the perception that information implicit in the equations of motion can be extracted without approximation using direct numerical simulation (DNS).

Direct numerical simulation of solid-liquid flows is a way of solving the initial value problem for the motion of particles in fluids. The particles are moved by Newton's laws under the action of hydrodynamic forces computed from the numerical solution of the fluid equations. To perform a direct simulation in the above sense, therefore, one must simultaneously integrate the NavierStokes equations (governing the motion of the fluid) and the equations of rigid-body motion (governing the motion of the particles). These equations are coupled through the no-slip condition on the particle boundaries, and through the hydrodynamic forces and torques that appear in the equations of rigid-body motion. These hydrodynamic forces and torques must of course be those arising from the computed motion of the fluid, and so are not known in advance, 
but only as the integration proceeds. It is crucial that no approximation of these forces and torques be made - other than that due to the numerical discretization itself - so that the overall simulation will yield a solution of the coupled initial value problem up to the numerical truncation error.

Many excellent numerical studies of particulate flows of many particles, which are not direct simulations in the above sense, have appeared in recent years. These approximate methods include simulations based on potential flow, Stokes flow, and point-particle approximations; they all simplify the computation by ignoring some possibly important effects like viscosity and wakes in the case of potential flow, inertial forces which produce lateral migration and acrossthe-stream orientations in the case of Stokes flow and the effects of stagnation and separation points in the case of point-particle approximations. Point particle approximations do advect particles by Newton's law but in some cases the influence of particles on the flow are neglected, in some cases the force on the fluid from the particles is added to the Navier-Stokes equations. Although this approach is often referred to as "direct simulations" by its practitioners, the forces on each particle are related to its motion and the fluid velocity by semi-empirical relations and this method is only applicable to dilute flows where there are no direct particle-particle interactions.

One goal pursued here is to demonstrate that numerical experiments on fluidization can be processed in log-log plots for straight lines leading to power laws as did Richardson and Zaki [1954] for real experiments. As far as we know, we are the only group of researchers to carry out this program. There is no prior literature, in which power laws are obtained from numerical experiments. On the other hand, there are a number of numerical packages for particles in fluids that might be used in this way. The methods of Stokesian dynamics (see Brady [1993]) can be recommended for problems in which inertia is absent. Following earlier work by Wachmann, Kalthoff, Schwarzer and Hermann [1998] and Wachmann and Schwarzer [1998], Hofler, Muller, Schwarzer and Wachmann [1999] introduced two approximate Euler-Lagrangian simulation methods for particle in fluids. In one method, the particle surface is discretitized in grid topology; spheres are polygons on flat places between nodes. In the second method, a volume force term is introduced to emulate rigid body motion on the particle surface; this method is similar to the force coupling methods, introduced by Maxey, Patel [1997]. Hofler, Muller, Schwarzer and Wachmann [1999] calculated sedimentation of 65,000 spheres but at Reynolds numbers so small that it is essentially Stokes flow. Johnson and Tezduyar [1999] used a fully resolved DNS/ALE method to compute sedimentation of 1,000 spheres at Reynolds numbers not larger than 10. A fully resolved method which is based on matching explicit Stokes flow representations of flow near particles with computations on a grid has been proposed by Ory, Oguz and Prosperetti [2000]. The problem of particulates in turbulent flows has been considered by a few authors: Crowe, Chung, Troutt [1996], McLaughlin [1994], Maxey, Patel, Chang, Wang [1997]; these approaches use point particle approximations because fully resolved computations in turbulent flow are not presently possible. Numerical approaches to particulate flow based on the discrete Lattice Boltzmann equation have been presented by Ladd [1994, 1996, 1997] Aidun [1996], Aidun, Lu and Ding [1998] and Qi [1997, 1999].

The correlations of Richardson and Zaki [1954] are an empirical foundation for fluidized bed practice. They did very many experiments with different liquids, gases and particles. They plotted their data in log-log plots; miraculously this data fell on straight lines whose slope and intercept could be determined. This showed the variables follow power laws. This same method 
works for numerical experiments on fluidization and on the lifting of particles across streamlines in Poiseuille flow (see Patankar, Ko, Choi, Joseph [2001], Patankar, Huang, Ko, Joseph [2001]). The existence of power laws can be regarded as a consequence of similarity Barenblatt [1996]; it is not an obvious consequence of the physics of flow of particulates or of the equations of motion. The possibility that power laws underlie the flows of dispersions generally could be considered.

In this article, we present the results of a simulation of the fluidization of 1204 spheres which are in satisfying agreement with results obtained by experiments. We will discuss numerical method briefly in Section 2 and then experiments in Section 3. In section 4 we present numerical results and compare those results with experimental ones in Section 5. Section 6 deals with the problem of computing time averages from computations. Section 7 examines the decomposition of the pressure gradient and specifies the variation with solids fraction of the part of the pressure gradient due to wall friction. The Richardson-Zaki correlation from DNS is discussed in Sections 8 and 9.

\section{Numerical Method}

To perform the direct numerical simulation of particulate flow, Glowinski et al [1997, 1998, 1999, 2001] have developed a methodology that is a combination of a distributed Lagrange multiplier based fictitious domain method (DLM) and operator splitting methods. The basic idea is to imagine that fluid fills the space inside as well as outside the particle boundaries. The fluidflow problem is then posed on a larger domain (the "fictitious domain"). This larger domain is simpler, allowing a simple regular mesh to be used. This in turn allows specialized fast solution techniques. The larger domain is also time-independent, so the same mesh can be used for the entire simulation, eliminating the need for repeated remeshing and projection (see Figure 1). This is a great advantage, since for three-dimensional particulate flow the automatic generation of unstructured body-fitted meshes in the region outside a large number of closely spaced particles is a difficult problem. In addition, the entire computation is performed matrix-free, resulting in significant savings. 


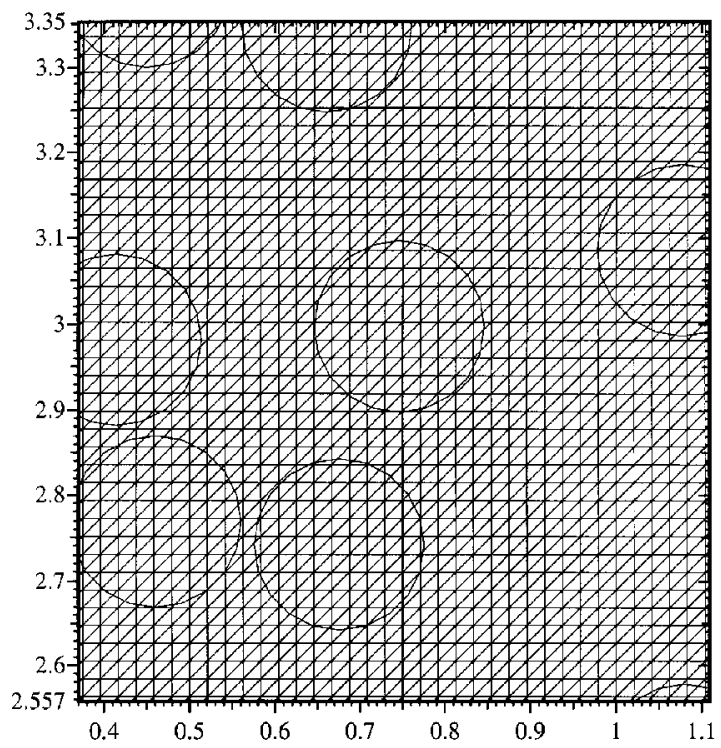

Figure 1. Part of a 2D example of a fixed triangular grid used in DLM computation. The same grid covers the fluid and solid. The fluid in the circle is bordered by Lagrange multipliers to move as a rigid body.

The velocity on each particle boundary must be constrained to match the rigid-body motion of the particle. In fact, in order to obtain a combined weak formulation with the hydrodynamic forces and torques eliminated, the velocity inside the particle boundary must also be a rigid-body motion. This constraint in enforced using a distributed Lagrange multiplier, which represents the additional body force per unit volume needed to maintain the rigid-body motion inside the particle boundary, much like the pressure in incompressible fluid flow whose gradient is the force required to maintain the constraint of incompressibility.

Concerning the space approximation of the problem by finite element methods, we use $P_{1}$-iso- $P_{2}$ and $P_{1}$ finite elements for velocity field and pressure respectively in the space approximation like in Bristeau, Glowinski and Periaux [1987]. Then we apply an operatorsplitting technique of Marchuk-Yanenko discussed in Marchuk [1990] for discretization in time. (Operating-splitting schemes have been used for solving the Navier-Stokes equations by many authors, starting, to our knowledge, with A. Chorin [1967, 1968, and 1973].) The linearly constrainted quadratic minimization problems which arise from this splitting are solved using conjugate gradient algorithms, yielding a method that is robust, stable, and easy to implement. For further details, see Glowinski, Pan, Hesla and Joseph [1999]. The immerse boundary methods of C. Peskin and his collaborators, Peskin [1977, 1981], Peskin and McQueen [1980], on the simulation of incompressible viscous flow in regions with elastic moving boundaries also uses a fictitious domain method, but without Lagrange-multipliers.

The statement that DNS fully resolves the solid-liquid flow should be qualified to say resolved up to the treatment of collisions. To prevent particles from penetrating each other or the walls, we adopt the following collision strategy. If we consider the particular case of circular particles in 2-D or spherical particles in 3-D, and if $B_{i}$ and $B_{j}$ are such two particles, with radiuses $R_{i}$ and $R_{j}$ and centers of mass $\vec{G}_{i}$ and $\vec{G}_{j}$, we shall require the repulsive force $\vec{F}_{i j}^{p}$ between $B_{i}$ and $B_{j}$ to satisfy the following properties: 


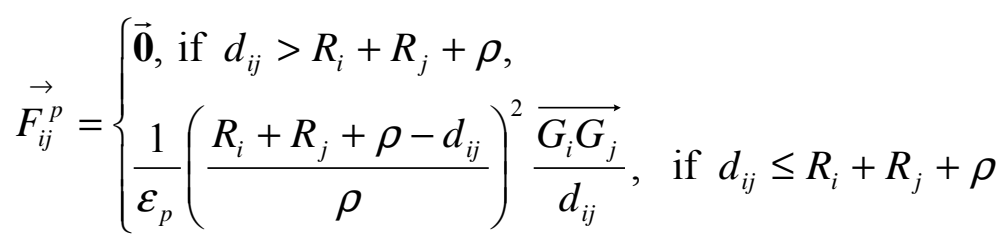

where $d_{i, j}=\left|\overrightarrow{G_{i} G_{j}}\right|, \rho$ is the force range, and $\varepsilon_{p}$ is a given small positive "stiffness" parameter chosen so that particles never touch.

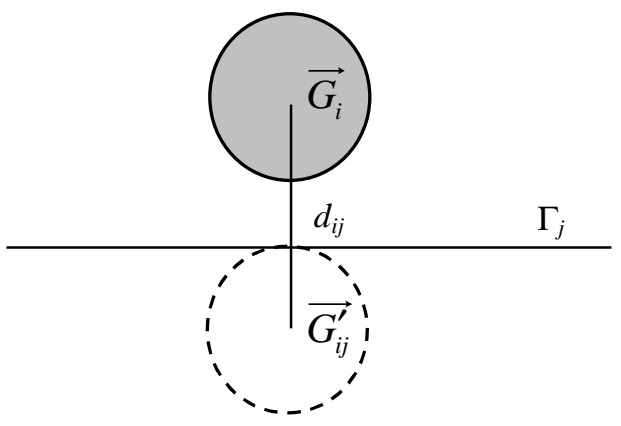

\section{Figure 2. Imaginary particle}

To treat particle-wall interactions we use a similar approach by introducing an imaginary particle as shown in Figure 2, where $d_{i j}=\left|\overrightarrow{G_{i} G_{i j}^{\prime}}\right|$. The choice of $\varepsilon_{p}$ is discussed in Glowinski, Pan, Hesla, Joseph, Periaux [2001, Section 5]. Indeed, the above repulsion force does not belong to the problem's description. It is invoked to keep the particles apart. Smooth particles should not collide, there is always a liquid film; if we do not provide for film rupture, the repulsive lubrication forces get larger and larger as the film gets smaller.

Fortunately the artificial repulsive force does not seem to have a big effect on the global motion (see Figure 5). However, the implementation of a security zone has the unfortunate consequence that particles cannot close pack. Though this does not seem to effect fluidized flows greatly, we must be able to generate close packing if we are able to accurately model the frictional resistances between close packed solids and walls.

We have developed but not yet implemented several methods in which repulsive forces are activated only when particles touch; this strategy will allow true tolerance of the mesh.

The DLM approach uses uniform grids for two and three-dimensional domains, and relies on matrix-free operations on the velocity and pressure unknowns in the domain. This simplifies the distribution of data on parallel architectures and ensures excellent load balance (see Pan, Sarin, Glowinski, Sameh and Periaux [1998]). The basic computational kernels, vector operations such as additions and dot products and matrix-free matrix-vector products, yield excellent scalability on distributed shared memory computers such as the SGI Origin 2000. A multilevel parallel elliptic solver, Sarin and Sameh [1998], has been incorporated into the DLM algorithm for twodimensional fluidized bed problems. This has yielded speedup of about 6 on 16 processors compared with the elapsed time on 2 processors on an SGI Origin 2000 at the NCSA. In addition, this represents an impressive eight-fold increase in speed over the best serial implementation. Even though there is a serial component of the 3D code, we have still observed that the speedup of 1.6 on 4 processors compared with the elapsed time on 2 processors on an 
SGI Origin 2000. But no more speedup can be gained if we increase to 8 processors from 4 processors. All numerical results reported in this article are obtained on 4 processors on an SGI Origin 2000 at the Minnesota Supercomputing Institute.

\section{Experiments}

We have carried out experiments of fluidization of 1204 spheres in a slit bed whose dimensions are

$$
\text { [depth, width, height }]=[0.686 \mathrm{~cm}, 20.30 \mathrm{~cm}, 70.22 \mathrm{~cm}] .
$$

The cross-sectional area of the bed is

$$
A=0.686 \times 20.30=13.32 \mathrm{~cm}^{2} .
$$

We could not measure variations of the gap size inside the bed, but the glass plates were pressured against $0.686 \mathrm{~cm}$ spacers by aluminum screw clamps. The nominal diameter of the spheres was

$$
d=0.635 \mathrm{~cm}(1 / 4 \text { inch }) .
$$

The sphere diameters varied from 0.635 to 0.6465 (see Figure 3). The averaged diameter of a sphere was

$$
\bar{d}=0.639826 \mathrm{~cm} .
$$

The density of the nylon sphere is

$$
\rho_{s}=1.14 \mathrm{gm} / \mathrm{cc} .
$$

The sphere was fluidized in water (we did not monitor the room temperature) whose density and viscosity are

$$
\rho_{f}=1 \mathrm{gm} / \mathrm{cc}, \quad \eta_{f}=0.01 \text { poise. }
$$




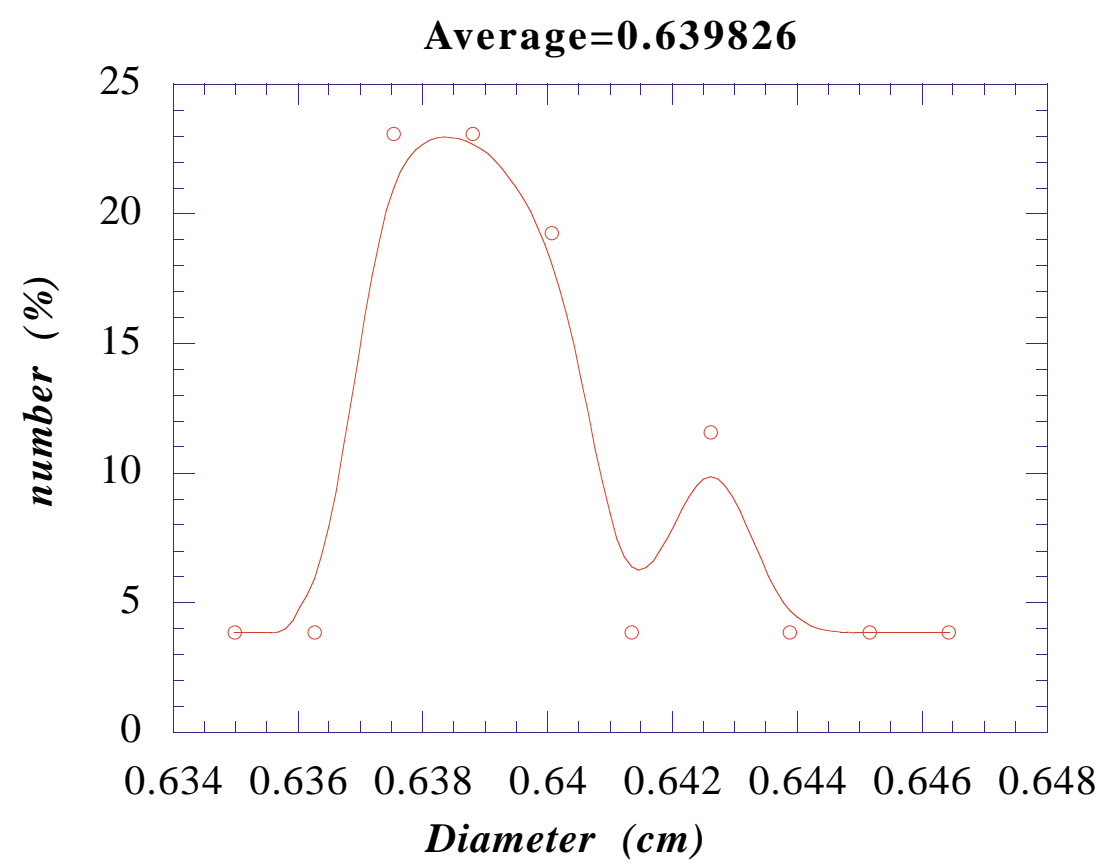

Figure 3. Distribution of diameter of spheres used in the fluidization experiment.

The Reynolds number based on the fluidization velocity for a single sphere is

$$
R=\frac{V(0) d}{\eta_{f} / \rho_{f}} \approx 730 .
$$

Local Reynolds numbers in a fluidized suspension can be larger because of the back flow through construction formed by nearly spheres. The velocity

$$
V_{2}=3.00 \mathrm{~cm} / \mathrm{sec}
$$

for incipient fluidization was identified roughly to within $0.1 \mathrm{~cm} / \mathrm{sec}$ as the value in which spheres more loosely packed in the fixed bed lifted slightly away from nearly spheres. According to the Richardson-Zaki formula (9.3), the velocity should vary between $V_{i}$ and $V(0)$.

$$
3=V_{i} \leq V(\phi) \leq V(0)=11.5 \mathrm{~cm} / \mathrm{sec} .
$$

Our experiments were consistent with this inequality.

The water is injected at the bottom of the bed through an array of plastic tubes under a distributor and an eddy dampening screen. The resulting fluidizing velocity is not uniform but there is no evidence of systematic anisotropy as the flow passes through the distributor screen. Large eddies of hydrodynamic origin just exactly like the one shown in the 1204 sphere animation on http://www.aem.umn.edu/Solid_Liquid_Flows/ are always present in the experiments. The fluidizing velocity is computed from values of the mass flow rate measured by collecting the weight of the overflow in a beaker over a fixed period of time. The mass flow rate is 


$$
\rho_{f} \dot{Q}=W / \Delta t
$$

where $Q$ is the volume flow rate; $W$ is the weight of fluid in the beaker collected over time $\Delta t$. The fluidizing (superficial) velocity is

$$
V(\phi)=\dot{Q} / A
$$

where $A$ is the cross-sectional area $13.32 \mathrm{~cm}^{2}$.

The height of the bed is measured by averaging the height of the top layer of particles. Stable bed heights with large fluctuations were typical. The measured values of the bed height as a function of the fluidization velocity $V(\phi)$ are presented in Figures 4 and 17 where they are compared with numerical simulation.

The solids fraction at a fluidization velocity $V(\phi)$ is given by inverting the height $H(\phi)$

$$
\phi=\frac{\Omega_{s}}{\Omega}=\frac{1204 \pi d^{3} / 6}{A H}=\frac{4.437}{H} .
$$

\section{Numerical Simulation}

The calculation was carried using the distributed Lagrange multiplier method (DLM) described in Section 2. The mesh size for velocity is

$$
h_{V}=0.06858 \mathrm{~cm}
$$

so the number of nodes is $3,348,675(11 \times 297 \times 1025)$. The mesh size for the pressure $h_{p}=2 h_{V}$ $(458,622)$ nodes. The time steps used in the computation are either 0.001 or 0.0005 seconds, with smaller time steps taken at times when the bed is fully expanded. The main parts of the computation were carried out with a partially parallel code; the computation time for running it is 115 seconds per time step on four R12000 processors in a SGI Orgin2000 at the Minnesota Supercomputing Institute. For example, the in case $V=4.5 \mathrm{~cm} / \mathrm{s}$, it took about 1660 hours to reach time $\tau=26$ seconds in the simulation; this is 63.84 hours of computation time for 1 second of real time.

In the simulation, the initial configuration of particles for the case of $V=3 \mathrm{~cm} / \mathrm{s}$ is a square lattice. The initial flow filed is zero everywhere for the case of $V=3 \mathrm{~cm} / \mathrm{s}$. Then we used the results of $V=3$ at $t=13$ (resp., $t=19.5$ ) as the initial conditions for the case of $V=3.5$ (resp., $V=2$ ). For the case of $V=4$ (resp., $V=4.5$ ), the initial conditions are obtained from the case of $V=3.5$ (resp., $V=4$ ) at $t=16.15$ (resp., $t=2$ ). And finally for the case $V=5$, the initial conditions are obtained from the case of $V=4.5$ at $t=27.2$. The above choices of initial conditions explains why the starting values of the bed height for different $V$ are different in Figure 4. For the parameters in (2.1) and (2.2), we took

$$
\varepsilon_{p}=5 \times 10^{-7}, \varepsilon_{w}=\varepsilon_{p}, \rho=h_{v}=0.06858 \mathrm{~cm} .
$$


The force range $0.06858 \mathrm{~cm}$ is larger than the distance $(0.686-0.635) / 2=0.0255 \mathrm{~cm}$ between a centered ball and a side wall. Hence, in the simulation the balls are effectively centered between the close walls by the particle-wall repulsive force. This centering mechanism is artificial; in the experiments the balls can go closer to one wall or another. Therefore, the drag on the balls in the experiment is larger than the drag in the simulation.

Figure 4 gives the bed height $H(t)$ as a function of time for different fluidizing velocities. The bed height is the average height of the top layer of spheres. The height rise curves have been extrapolated to terminal rise for large times by a least square fit to $a+b \exp \{-c t\}$. For the case $V=3 \mathrm{~cm} / \mathrm{s}$ we fit $H(t)$ to $a+b \exp \{-c(t-2)\}$ for $t>2$ because the bed height first decreases. The terminal values

$$
a=\lim _{t \rightarrow 0} H(t)
$$

are given by

\begin{tabular}{|c|c|}
\hline $\boldsymbol{V}(\mathbf{c m} / \mathbf{s})$ & $\boldsymbol{a}$ (inches) \\
\hline 3 & 13.33 \\
\hline 3.5 & 16.84 \\
\hline 4 & 19.10 \\
\hline 4.5 & 21.29 \\
\hline 5 & 25.52 \\
\hline
\end{tabular}




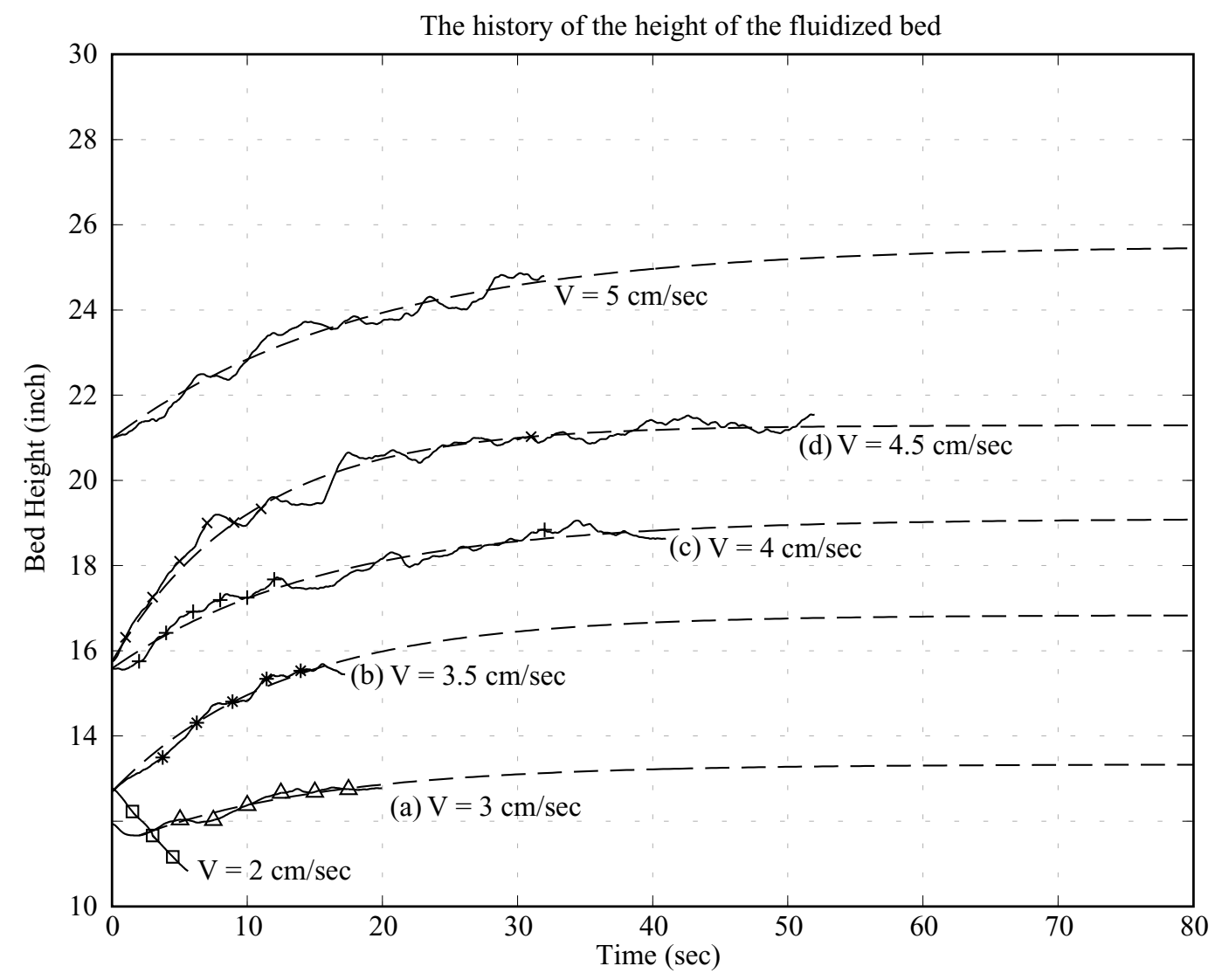

Figure 4. Bed height $H$ vs. time for different values of the fluidizing velocity $V . H$ is the average height of the top layer of 1204 spheres. The letters $(a) \ldots(d)$ index the snapshots shown in Figures 9-10. The dashed lines - - are least square fits to $H(t)=a+b \exp (-c t)$.

To test the effect of changing the size of the security zone in a relatively short time we studied the fluidization of 150 spheres rather than 1204 spheres. The size of the security zone was reduced to $h_{v} / 2$. Figure 5 shows that the change in the height rise is modest, the smaller security zone allows for an increase in the bed height of about 2 or 3 percent. 


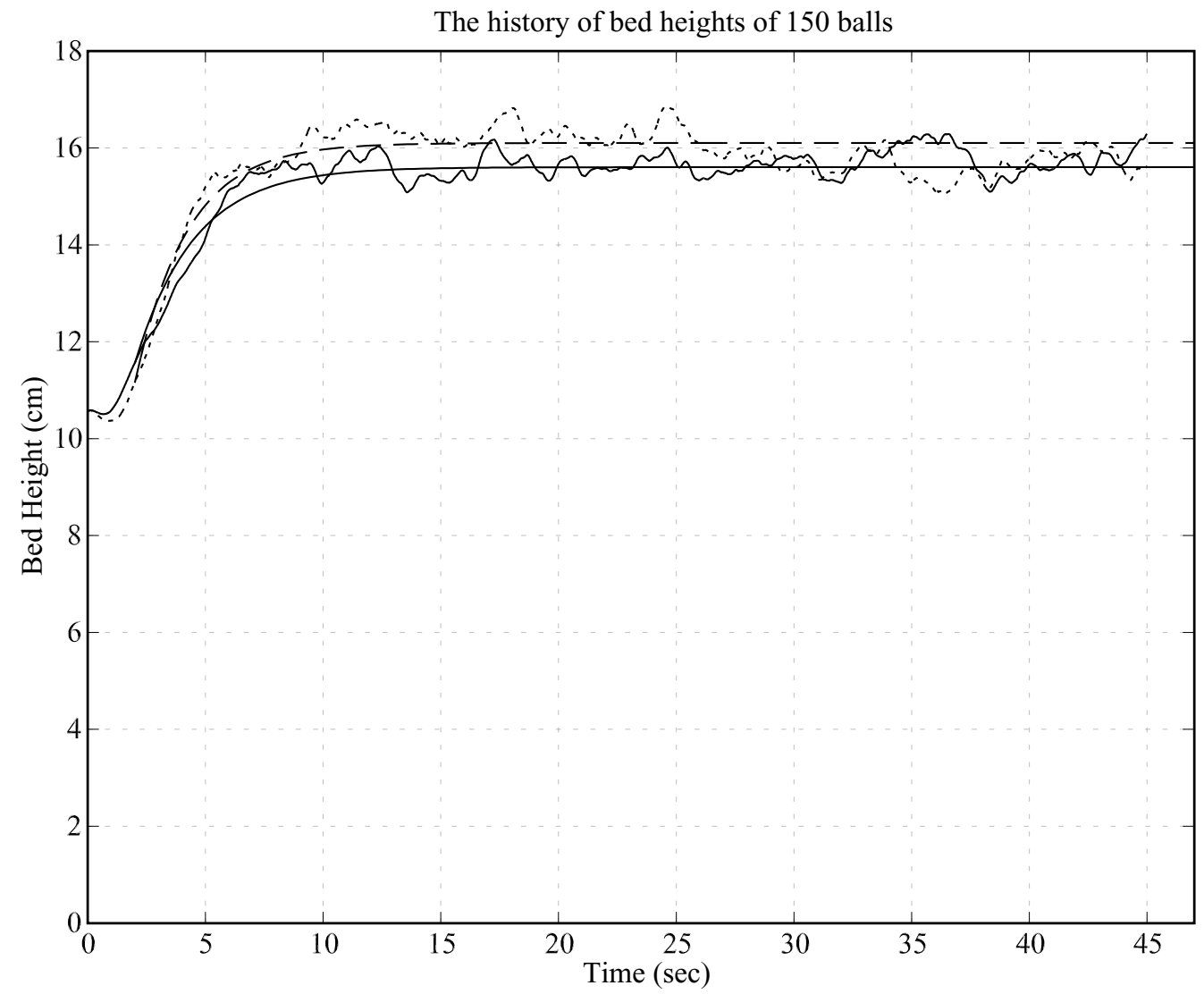

Figure 5. Bed height $H$ vs. time for $V=4 \mathrm{~cm} / \mathrm{s} . H(t)$ is the average height of the top layer of 150 spheres. The lines are least square fits to $H(t)=a+b \exp (-c(t-2))$. Dashed - - security zone 0.5 $h_{v}$; solid - security zone $h_{v}$.

\section{Qualitative comparison of experiment and simulation}

The simulation was carried out in a fluidization column whose coordinates are shown in Figure 6.

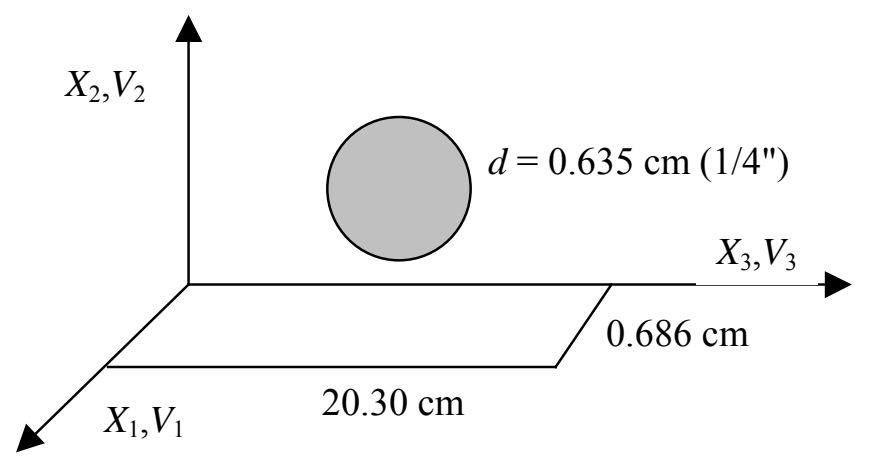

Figure 6. Coordinates and velocity components in the fluidization column. The fluidization velocity is $V \equiv V_{2}$.

In Figures 7 and 8 snapshots of simulation of 1204 spheres are shown in perspective to emphasize that the simulation is three-dimensional. The video animations of these simulations, 
which can be found at www.aem.umn.edu/Solid-Liquid_Flows, cannot be distinguished from real experiments. In Figures 9-10 we compare snapshots of simulations in frontal view with snapshots from experiments under equivalent conditions. We also compare snapshots of simulations in frontal view of $V=4 \mathrm{~cm} / \mathrm{s}$ with snapshots from experiments under equivalent conditions for the case of $V=4.037 \mathrm{~cm} / \mathrm{s}$ in Figures 11 and 12 . We can find that the simulation results do have features shown in the snapshots from experiments.
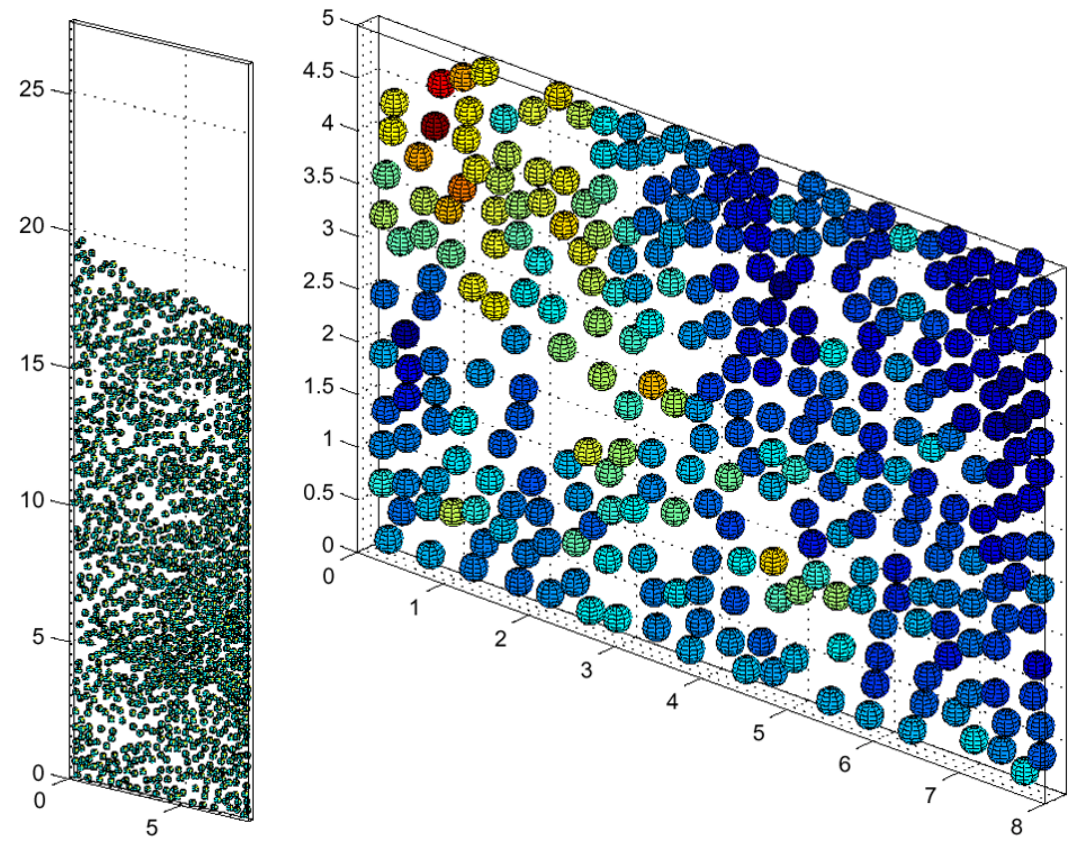

Figure 7. Snapshot of simulation of 1204 spheres with $V=4$ (left) and a blow-up (right) at $t=32$.
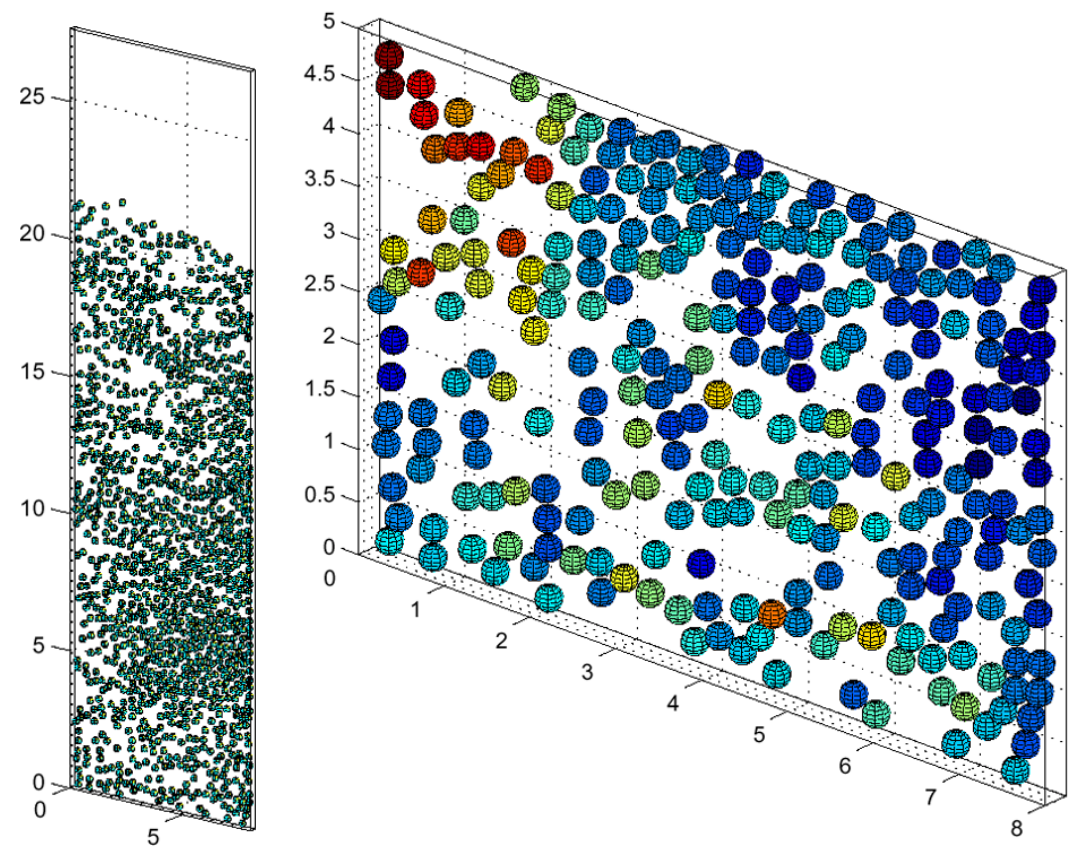

Figure 8. Snapshot of simulation of 1204 spheres with $V=4.5$ (left) and a blow-up (right) at $t=31$. 

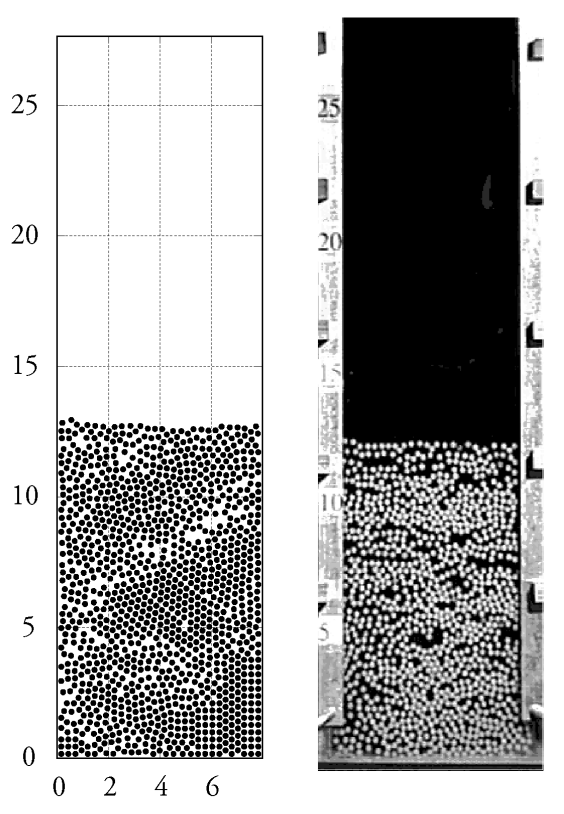

- $\mathrm{V}=3$ : Particle position at $\mathrm{t}=20$.

- The maximal particle Reynolds number is 1142 .

- The maximal averaged particle Reynolds number is 131.
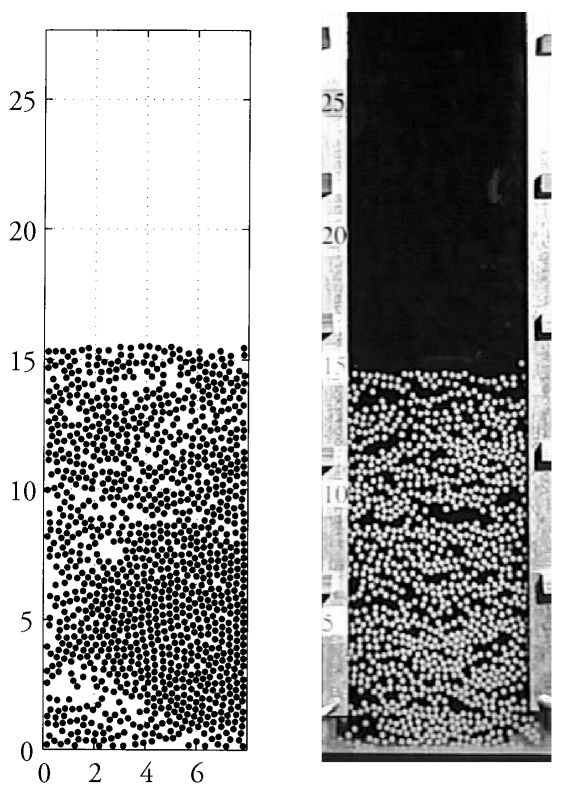

- $\mathrm{V}=3.5$ : Particle position at $\mathrm{t}=17.238$

- The maximal particle Reynolds number is 1671 .

- The maximal avaraged particle Reynolds number is 236 .

Figure 9. Cf. (a) in Figure 4 (left); $c f$. (b) in Figure 4 (right).
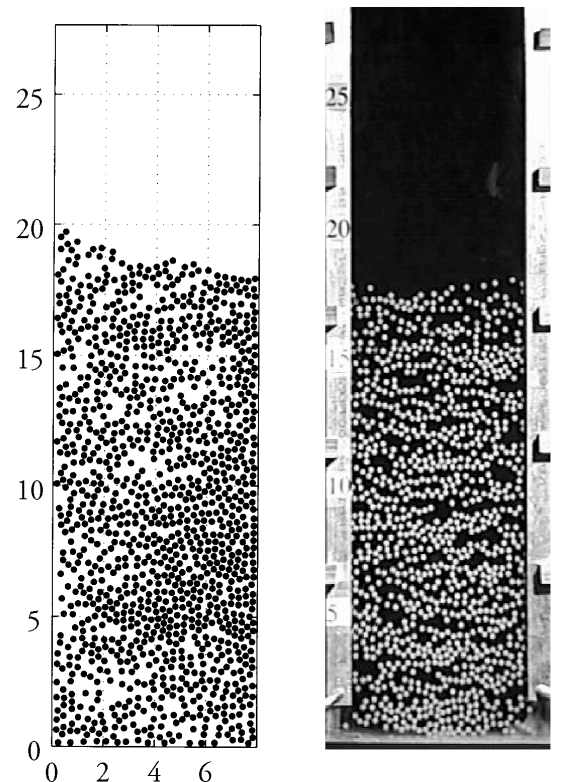

- $\mathrm{V}=4$ : Particle position at $\mathrm{t}=32$.

- The maximal particle Reynolds number is 1965.

- The maximal avaraged particle Reynolds number is 276 .
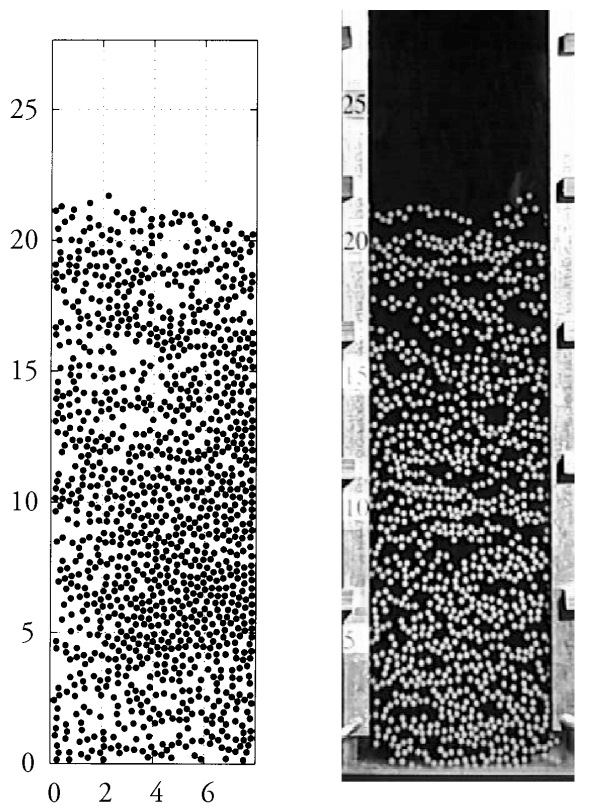

- $\mathrm{V}=4.5$ : Particle position at $\mathrm{t}=31$.

- The maximal particle Reynolds number is 1859 .

- The maximal averaged particle Reynolds number is 292.

Figure 10. Cf. (c) in Figure 4 (left); $c f .(d)$ in Figure 4 (right). 

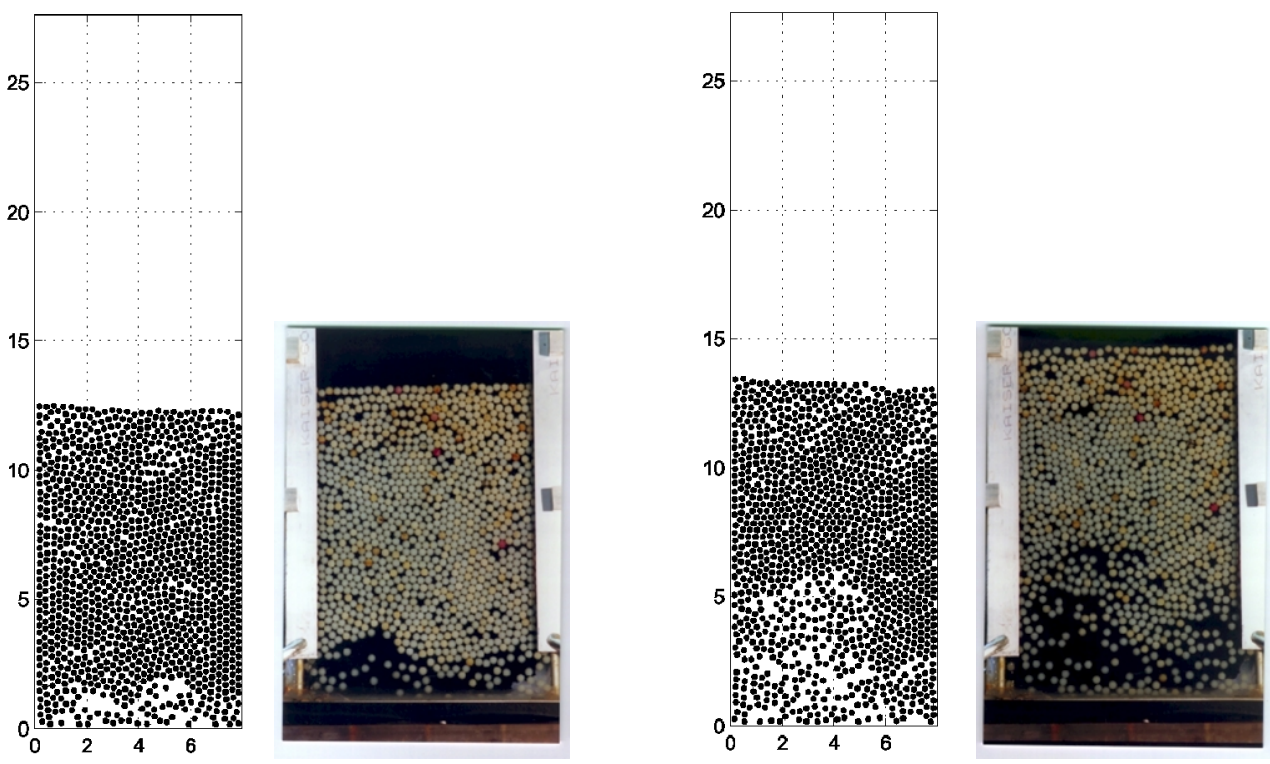

Figure 11. Comparison of snapshots of simulations of $V=4$ at $t=2$ (left) and 4 (right), and snapshots from experiments under equivalent conditions for the case of $V=4.037$.
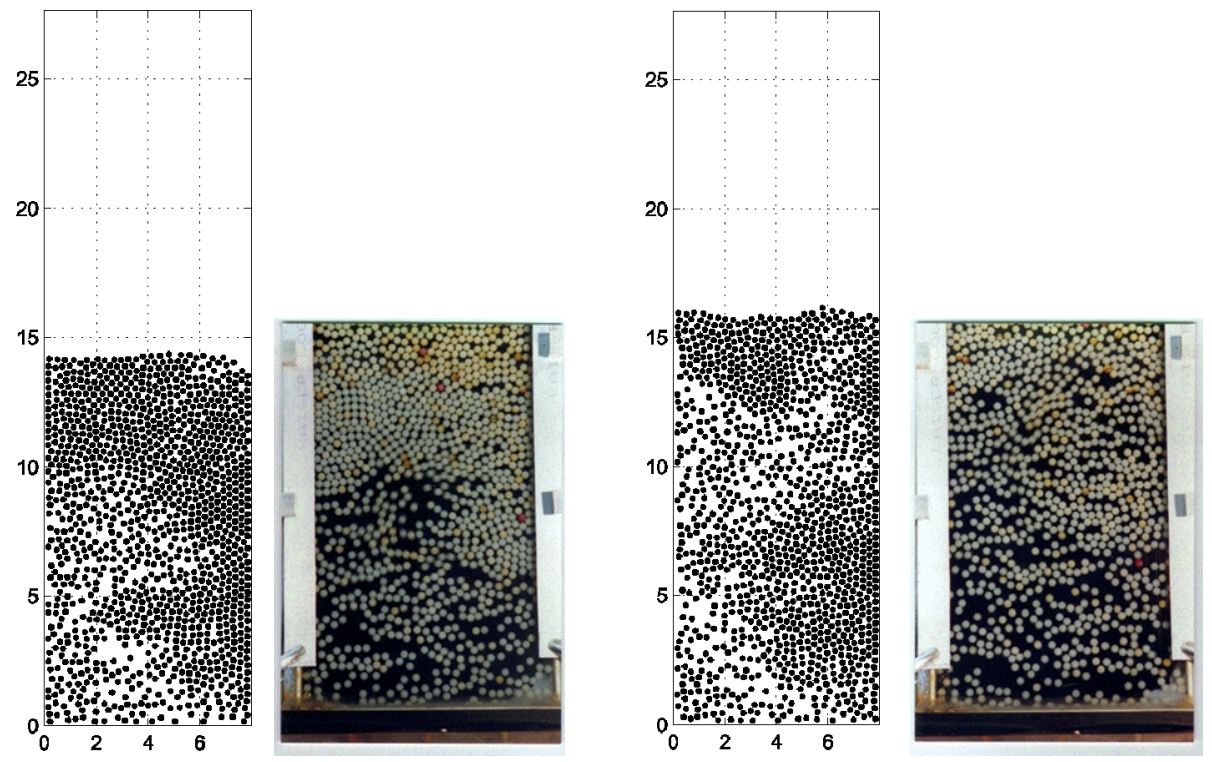

Figure 12. Comparison of snapshots of simulations of $V=4$ at $t=6$ (left) and 9 (right), and snapshots from experiments under equivalent conditions for the case of $V=4.037$.

\section{Numerical computation of averaged quantities}

DLM produces huge amounts of data at each one of millions of nodes. The problem is how to structure this data to extract useful information; we must decide beforehand what data to collect as values to store for post processing. The fixed node property of DLM is particularly adapted to the collection of data in a form suitable for averaging methods used to construct models. 
To define a data structure we define a data string; this is a sequence of numbers produced at that node. We call the number of values in the data string the number of hits. In these simulations in which time steps are $\Delta t=0.001 \mathrm{sec}$ for start up and $0.0005 \mathrm{sec}$ for the later times we may get 10,000 hits or more in a long simulation. Sometimes a particle is at the node, at the other times fluid is there. By processing data at hits we can create time averages without significant cost in computational cost. Suppose a solid is at the node $M$ times and the fluid is there $M^{\prime}$ times, $M+M^{\prime}=10^{4}$. Then $\phi=M / 10^{4}$ and $\varepsilon=M^{\prime} / 10^{4}, \varepsilon+\phi=1$ gives the volume fractions. If $V(\mathbf{x}, t)$ is the component of velocity parallel to gravity and $\mathbf{x}$ is at a node and $V_{l}(\mathbf{x}, t)$ are a data string of hits. Then

$$
V_{s}(\mathbf{x}, t)=\frac{1}{M} \sum_{i=1}^{M} V_{i}(\mathbf{x}, t)
$$

where $\mathbf{x}$ is at a node; obviously

$$
V_{f}(\mathbf{x}, t)=\frac{1}{M^{\prime}} \sum^{M^{\prime}} V_{i}(\mathbf{x}, t)
$$

Then, at the same node, we ought to find

$$
V(\phi)=V_{s} \phi+V_{f} \varepsilon
$$

After transients have disappeared in the fluidization we ought to find that $V_{s}=0$.

In the same way, we can find that a data string of values of the angular velocity as a difference of the velocity at a point in the solid and its mass center and so on for other averages.

The time averages formed from data strings can be thought to be ensemble averages on nontransient flow and may be assumed to be local time averages on intervals with a sufficiently large number of hits which is small relative to the length of time of transients. By repeating simulations we could actually initiate the procedure used to generate ensemble averages. There is a mathematical literature on the relation of time averages to ensemble averages which is rather theoretical and involves assumptions of a mathematical nature which are difficult to verify.

In our simulation of 1204 spheres we created such data strings at 38 nodes on a line across the width of the bed at a height of $10.179 \mathrm{in}$. in a plane in the center of the depth, $0.135 \mathrm{in}$. from each wall. The width of the bed is 8 in. and the 38 nodes plus 2 wall points means that the nodes are separated by $1 / 10$ in. The number of hits was $M=7365$ for the case $V=4.5 \mathrm{~cm} / \mathrm{sec}$ taken every 4 time steps. In Figure 13 we present the solid fraction $\phi$ and the three components of the average solid $V_{s}$, liquid $V_{f}$ and composite velocity $V(\phi)=V_{s} \phi+V_{f}(1-\phi)$ for the case $V=4.5 \mathrm{~cm} / \mathrm{s}$. The velocity components $V_{1}, V_{2}$ and $V_{3}$ correspond to coordinates in the cross section of the fluidization column shown in Figures 13(b) - 13(d).

The data presented in Figure 13 shows that the dynamics of the bed are strongly twodimensional, the velocity component $V_{2}$ is in the vertical direction; in the one-dimensional approximation the composite velocity

$$
V_{2 C}(\phi)=V_{2 S}(\phi)+V_{2 f}(1-\phi)
$$


would equal $V(\phi)$. Obviously $V_{2}=V_{2}\left(x_{3}\right)$ because of a large circulating eddy which is apparent on the video animations on http://www.aem.umn.edu/Solid-Liquid_Flows and in experiments. It is also apparent that the difference between averaged fluid velocity $V_{2 f}$ and the averaged solid velocity $V_{2 S}$ is positive; the solid lags the fluid by 3 to $5 \mathrm{~cm} / \mathrm{s}$. The transverse components of velocity $V_{1}$ and $V_{3}$ are basically zero which is an assumption one makes in a one-dimensional theory. The fluctuation level of $V_{1}$ is very low because of the collision strategy interacting with nearby side walls, but the particles are not rigorously centered by these artificial collision forces.

It would be desirable to have many more points in our data strings. Greater computational efficiency and speed is a challenge for the future.

Figure 13 shows a strong two-dimensional variation of average equations across the slit column which is suppressed in one dimensional studies.
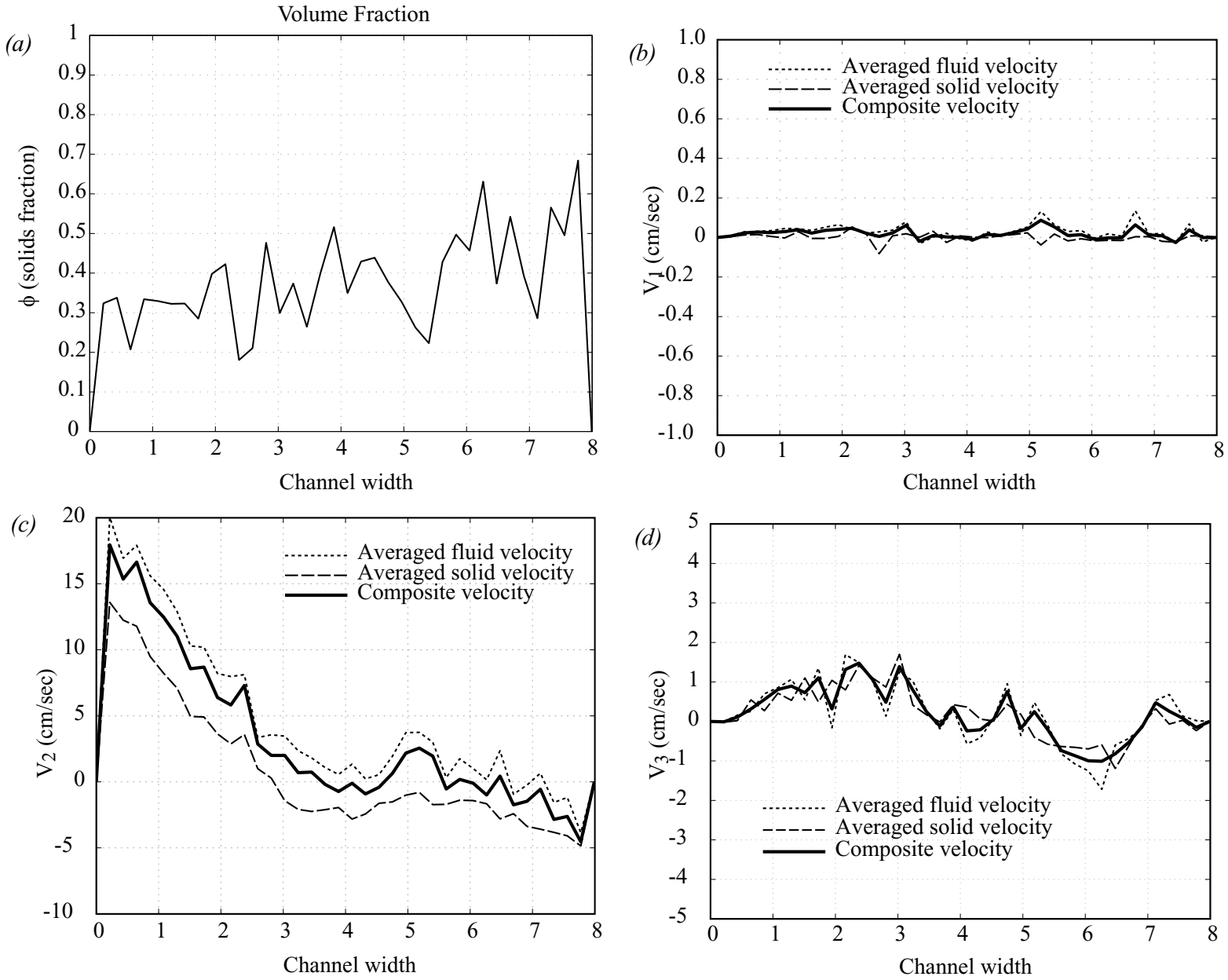

Figure 13. Volume fraction $\phi$ and averaged components of velocity $\mathrm{V}_{1}, \mathrm{~V}_{2}, \mathrm{~V}_{3}$ in coordinate direction $\mathrm{x}_{1}$, $\mathrm{x}_{2}, \mathrm{x}_{3}$ at 38 nodes spaced at 1/10 in. across the 8 in. side of the fluidization column when $\mathrm{V}=4.5$ $\mathrm{cm} / \mathrm{s}$. (a) $\phi$, , (b) $\mathrm{V}_{1}$, (c) $\mathrm{V}_{2},(d) \mathrm{V}_{3}$. The difference between the averaged fluid and averaged solid velocity is the slip velocity. This is the first exact calculation of the slip velocity in a fluidized suspension. 


\section{Dynamic and wall friction pressure in a fluidized bed}

The stress in an incompressible Newtonian fluid is given by

$$
\boldsymbol{\sigma}=-P \mathbf{1}+2 \mu \mathbf{D}[\mathbf{u}]
$$

where $\mathbf{D}[\mathbf{u}]$ is the symmetric part of the velocity gradient $\nabla \mathbf{u}$ and $P$, the total pressure, is the mean normal stress. The equation of motion in the $z$ direction is

$$
\rho \frac{d w}{d t}=-\frac{d p}{d z}+\mu \nabla^{2} w
$$

where $w=\mathbf{e}_{z} \cdot \mathbf{u}$ and $p=P+\rho_{f} g z$ is the dynamic pressure that we compute in our DNS simulation.

We may compute an average dynamic pressure gradient $\frac{d \bar{p}}{d z}$ by averaging over cross sections and time. This quantity then is given by DNS and

$$
\frac{d \bar{p}}{d z}=\frac{d \bar{P}}{d z}+\rho_{f} g
$$

We may decompose

$$
\bar{p}=\bar{p}_{w}+\bar{p}_{s}
$$

where $\bar{p}_{w}$ is the wall friction pressure and $\bar{p}_{s}$ is the pressure required to fluidize the spheres. In fluidized bed practice it is assumed that when wall friction is negligible the pressure gradient

$$
\frac{d \bar{P}}{d z}=-\left(\rho_{P} \phi+\rho_{f} \varepsilon\right) g=-\rho_{c} g
$$

balances the composite weight of fluid plus solids. In this case

$$
\frac{d \bar{P}}{d z}=\frac{d \bar{p}}{d z}-\rho_{f} g=\frac{d \bar{p}_{s}}{d z}-\rho_{f} g=-\rho_{c} g
$$

Hence

$$
\frac{d \bar{p}_{s}}{d z}=-\left(\rho_{P}-\rho_{f}\right) g \phi
$$

This expression was verified in experiments of Wilhelm and Kwauk [1948] and Lewis, Gilliand and Bauer [1949]. Combining now (7.4) and (7.7) we get

$$
\frac{d \bar{p}}{d z}=\frac{d \bar{p}_{w}}{d z}-\left(\rho_{P}-\rho_{f}\right) g \phi
$$

When $\phi=0$, the dynamic pressure is equal to the wall friction pressure. 
Equation (7.8) is an equation for the wall friction pressure gradient with values of $d \bar{p} / d z$ and $\phi$ given by DNS. Table 1 gives the values of terms in this equation for different fluidizing velocities. The table shows that the wall friction pressure gradient is about $1 / 4$ of the pressure gradient needed to fluidize the spheres. As $V$ increased, $\phi$ decreases and the pressure gradient $-\left(\rho_{P}-\rho_{f}\right) g \phi$ to fluidize spheres decreases. The small decrease in the wall friction pressure gradient with fluidizing is surprising; the wall friction should go up as the speed increases. We conjecture that the decreased friction is due to a decrease in the effective viscosity $\mu(\phi)$ of the mixture as $\phi$ is decreased; the viscosity of densely packed mixtures is greater.

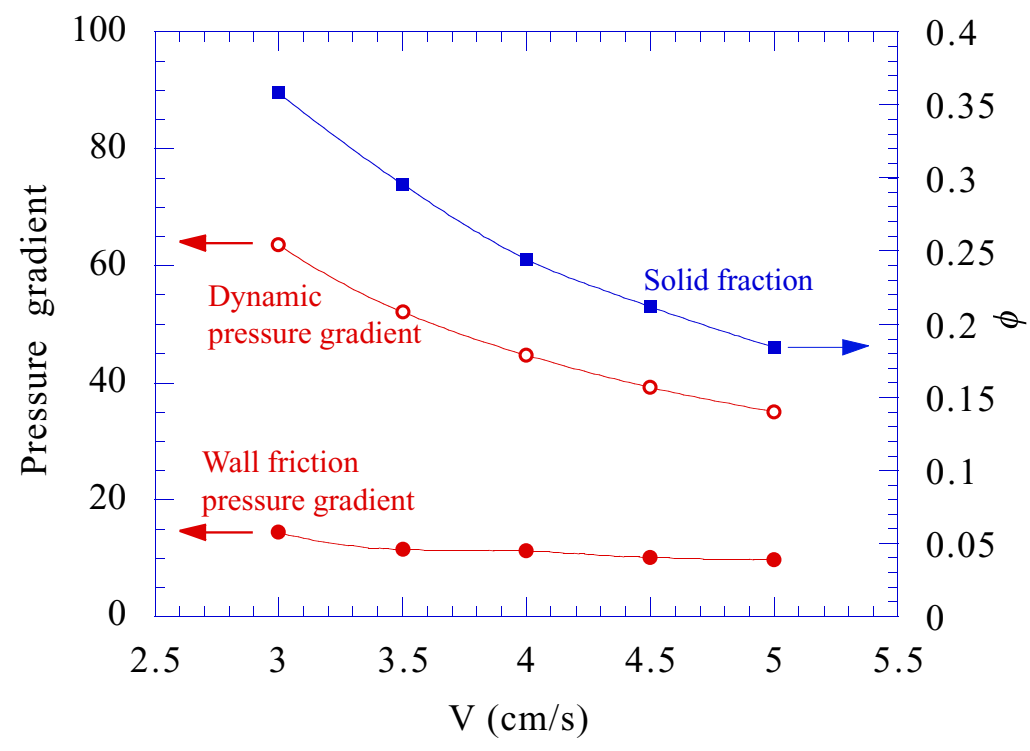

Figure 14. Solid fraction $f$ dynamic pressure gradient and wall friction pressure gradient vs. fluidizing velocity.

The calculation given here points a new direction for the interrogation of DNS for new results in continuum engineering descriptions. Our conclusions are tentative because the results about the wall friction pressure gradient depend on the accuracy of our numerical simulation near walls. It is certainly true that the activation of a repelling force when the particle approaches the wall reduces accuracy. We are confident that the size of the security zone can be reduced to zero by techniques under development so the full hydrodynamics of lubricating flow can be captured up to mesh resolution.

Table 1. Terms in the dynamic pressure gradient equation (7.8). $\rho_{p}=1.14 \mathrm{~g} / \mathrm{cc}, \rho_{f}=1 \mathrm{~g} / \mathrm{cc}$.

\begin{tabular}{|c|c|c|c|c|c|}
\hline$V(\mathrm{~cm} / \mathrm{s})$ & $-\frac{d \bar{p}}{d z}\left(\right.$ dynes $/ \mathrm{cm}^{2)}$ & $\phi$ & $\rho_{c} g$ & $\left(\rho_{p}-\rho_{f}\right) g \phi$ & $-\frac{d \bar{p}_{w}}{d z}=-\frac{d \bar{p}}{d z}-\left(\rho_{p}-\rho_{f}\right) g \phi$ \\
\hline 3 & 63.570 & 0.3582 & 1050.1 & 49.177 & 14.393 \\
\hline 3.5 & 52.050 & 0.2956 & 1021.2 & 41.246 & 11.463 \\
\hline 4 & 44.694 & 0.2439 & 1014.2 & 34.155 & 11.202 \\
\hline 4.5 & 39.171 & 0.2119 & 1009.8 & 29.758 & 10.076 \\
\hline 5 & 34.990 & 0.1842 & 1006.0 & 25.957 & 9.705 \\
\hline
\end{tabular}




\section{Sedimentation and fluidization velocity of single spheres}

We did simulations and experiments of the sedimentation and fluidization velocity of single spheres. It is sometimes assumed that these two velocities are the same but in general this is true only in very special cases.

The flow of fluid up the slit bed is close to a developing Poiseuille flow in which the velocity of the fluid vanishes at the wall but not at the center. In the sedimentation case the fluid does not move unless disturbed by a falling particle. The flow of fluid in a fluidized bed need not be fully developed. The flow profile can change from station to station in the fluidized bed case, but not in the sedimentation case.

In the ideal case there is one and only one fluidization velocity and, if we ignore the flow variations just mentioned, this velocity is the sedimentation velocity; in both cases the drag balances the buoyant weight. For steady flow fluidized and sedimenting particle velocities are equivalent under Galilean transformation. In fact we do not verify this ideal case in sedimentation or fluidization; a unique velocity does not emerge as can be seen from the experimental results given in Figure 15.

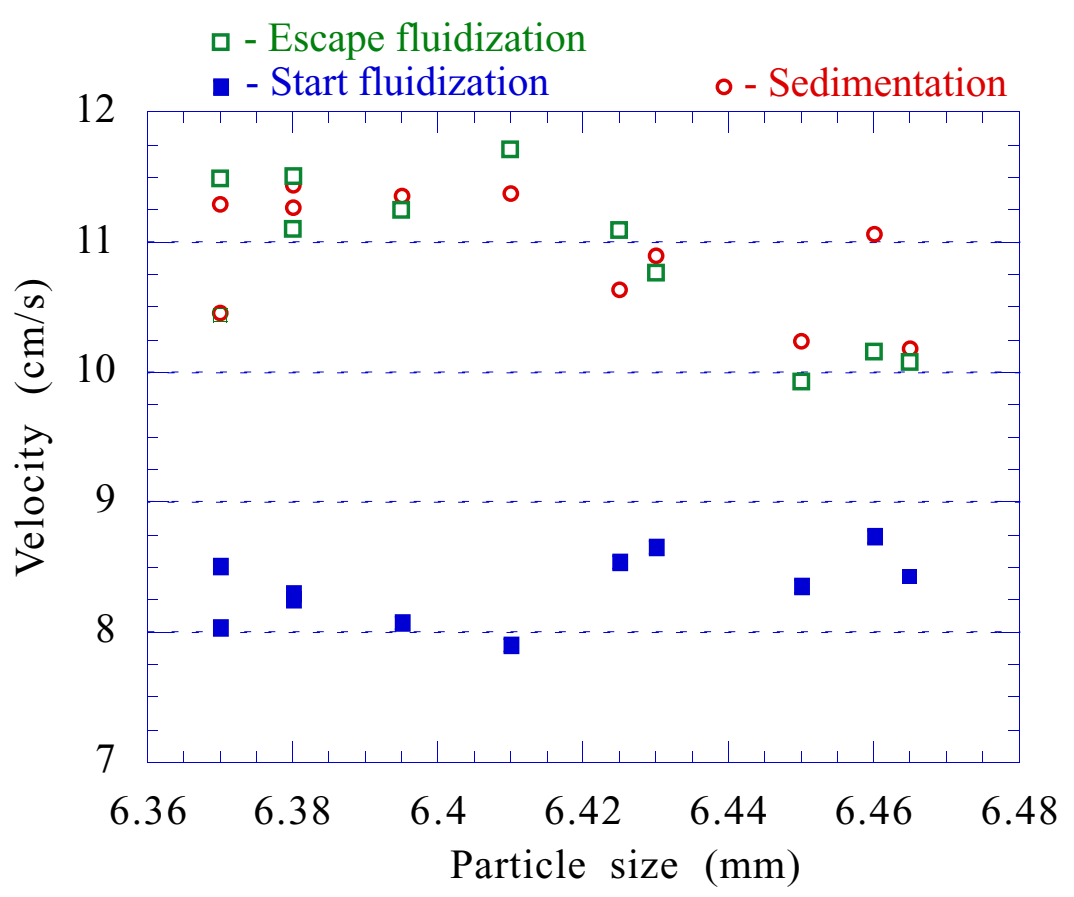

Figure 15. Sedimentation $\bullet$ and fluidization velocities $\mathbf{\square}$ from experiments using different size spheres.

The particle lifts off the distributor when $V$ is greater than $\mathbf{\square}$ and is dragged out of the column only when $V$ is greater than $\square$.

In Figure 15 we have plotted fluidization and sedimentation velocities for different spheres. Focusing first on sedimentation we note that even when we drop the same sphere in the quiet slit bed, the sedimentation velocity differs from trial to trial. Variations of as much as $7 \%$ are observed. How do we account for such variations? 
As a practical matter our slit bed has a nominal gap size of $0.686 \mathrm{~cm}$. The gap size is certainly not uniform; perhaps the gap size varies between 0.6778 and $0.7239 \mathrm{~cm}$. Obviously this variation will lead to a variation in the sedimentation velocity.

A more fundamental reason for the variability in the sedimentation rate is that the motion at Reynolds numbers in the thousands is not steady and is probably chaotic in the sense of dynamical systems due to vortex shedding. The falling spheres do not center rigorously but are in some kind of unsteady off-center motion that is not well understood. How and why this kind of unsteadiness leads to different settling velocities is not understood.

Table 2 lists the values of the velocity of sedimenting spheres of different diameter falling in a $0.686 \mathrm{~cm}$ gap between the walls of the sedimentation column. The sedimentation velocity appears to increase rather markedly with mesh refinement; extrapolated to a fine mesh $h \rightarrow 0$ would seem to imply a fall of about $10 \mathrm{~cm} / \mathrm{s}$ rather than the $8.7 \mathrm{~cm} / \mathrm{s}$ value observed in the smaller mesh. We do not see a consistent variation with diameter. This may be due to some kind of unsteadiness to which we alluded in the previous paragraph. There is a discrepancy between the values in Table 2 and those reported in the experiments of between $10 \%$ and $20 \%$ if $8.7 \mathrm{~cm} / \mathrm{s}$ is taken as the representative simulation value. We think that it is probable that the faster fall velocity in the experiments may be due to channeling through places where the gap size is larger.

Table 2. Averaged vertical terminal velocities of balls of different diameter and $\rho_{p}=1.14 \mathrm{~g} / \mathrm{cc}$ computed by DNS in the volume of Figure 6.

\begin{tabular}{|l|c|c|c|c|}
\cline { 2 - 5 } \multicolumn{1}{c|}{} & \multicolumn{4}{c|}{ Diameter } \\
\cline { 2 - 5 } \multicolumn{1}{c|}{} & $\mathbf{0 . 6 3 0 0} \mathbf{c m}$ & $\mathbf{0 . 6 3 5 0} \mathbf{c m}$ & $\mathbf{0 . 6 3 9 8 c m}$ & $\mathbf{0 . 6 5 0 0 c m}$ \\
\hline $\boldsymbol{h}=\mathbf{0 . 0 2 7}$ & -8.085378 & -8.130019 & -8.155760 & -7.790286 \\
\hline $\boldsymbol{h}=\mathbf{0 . 0 1 8}$ & -8.722692 & -8.738451 & -8.689694 & -8.724728 \\
\hline
\end{tabular}

The fluidization results are of great interest. We do not get a unique fluidization velocity; for each sphere there is a rather large interval of velocities for which the sphere does not fall to the bottom or blow out of the bed; the interval may range, say from $6 \mathrm{~cm} / \mathrm{s}$ to $11 \mathrm{~cm} / \mathrm{s}$. In fluidization velocities less than, say $6 \mathrm{~cm} / \mathrm{s}$ the sphere will not rise and for large velocities, say about $11 \mathrm{~cm} / \mathrm{s}$ the sphere will blow out of the bed.

An important and practically useful result arising from this study of fluidization is that the height of the sphere above the bottom increases with the fluidizing velocity. This positioning property is such that the position of the particle in the bed may be controlled at the dial setting of the inlet flow rate. The positioning property also arises form our simulation and is clearly evident in the rise curves shown in Figure 16. We have seen such positioning hydrodynamics in experiments on the fluidization of sensors in round pipes where the ability to position the sensor is of practical importance. 
The history of the height of a fluidized ball

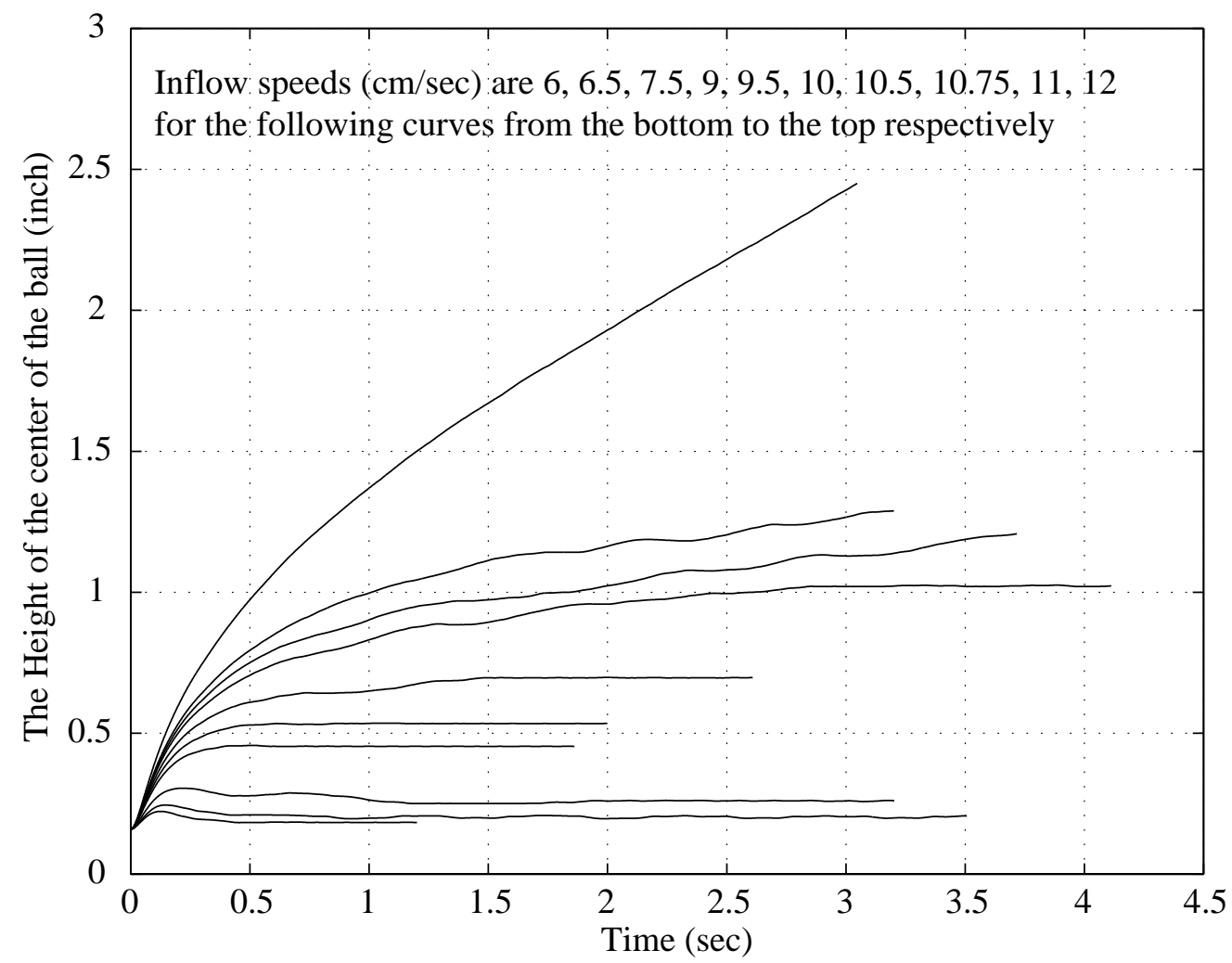

Figure 16. $\mathrm{H} v \mathrm{~s} . \mathrm{t}$ for a single particle $\mathrm{d}=0.635 \mathrm{~cm}$ from numerical simulation. $F$ or $\mathrm{V}<\mathrm{V}_{\mathrm{m}} \approx 6 \mathrm{~cm} / \mathrm{s}$ the sphere remains at the distributor; for $\mathrm{V}>\mathrm{V}_{\mathrm{m}} \approx 10.5 \mathrm{~cm} / \mathrm{s}$ the sphere is dragged out of the bed.

The aforementioned positioning hydrodynamics is not understood. A promising explanation follows from the observation that the average distance between the particle and the wall is a function of the flow speed. Loosely described, we could say that the Segré-Siberberg position of equilibrium between the centerline of close walls and the walls is a function of flow speed. The overall drag on the sphere, which in any case must balance the buoyant weight, is a function of flow speed and particle position. Evidently the flow speed produces a particle position such that the drag-weight balance is preserved at different heights in the bed.

In the simulation an exactly zero velocity of the sphere was not achieved. For $V \leq 6 \mathrm{~cm} / \mathrm{s}$ the particle will not rise. The particle velocity in the interval of fluidization is very nearly zero and rather random as shown in table 3 . The particle rises out of the bed when $V>10.5$. 
Table 3. The averaged vertical velocities of a ball of diameter $0.635 \mathrm{~cm}$ fluidized in a $2 D$-like bed of dimension $D \times W \times H=0.27^{\prime \prime} \times 7.992^{\prime \prime} \times 8.1^{\prime \prime}$ are in the following table. The ball was initially located at the center of the bottom of the bed.

\begin{tabular}{|c|c|}
\hline $\begin{array}{c}\text { In-flow velocity } \\
(\mathrm{cm} / \mathrm{s})\end{array}$ & $\begin{array}{c}\text { Averaged vertical speed } \\
\text { (after stabilized) }\end{array}$ \\
\hline 6 & -0.00522 \\
\hline 6.5 & 0.00827 \\
\hline 7.5 & 0.00219 \\
\hline 9 & -0.00178 \\
\hline 9.5 & 0.00631 \\
\hline 10 & 0.0006997 \\
\hline 10.5 & 0.00521 \\
\hline 10.75 & 0.276 \\
\hline 11 & 0.265 \\
\hline 12 & 1.260 \\
\hline
\end{tabular}

\section{Richardson-Zaki correlations from DNS}

Here we introduce an application of DNS that we call the method of correlations. The method is inspired by the work of Richardson and Zaki [1954]. They processed their data in loglog plots and found straight lines leading to power laws. The method of correlations follows the same procedure using numerical data from DNS rather than experimental data. Data from our real and numerical experiments are shown in Figure 17. The experiment and simulations do not quite match. The 1204 spheres used in the experiments are polydisperse (Figure 3) with an average diameter of $0.6398 \mathrm{~cm}$ rather than the $0.635 \mathrm{~cm}$ diameter used in the simulation. Moreover the lowest data point for the simulation may be inaccurate because the artificial repulsive force which is activated to keep particles apart makes accurate calculations near close packing at incipient fluidization less accurate. 


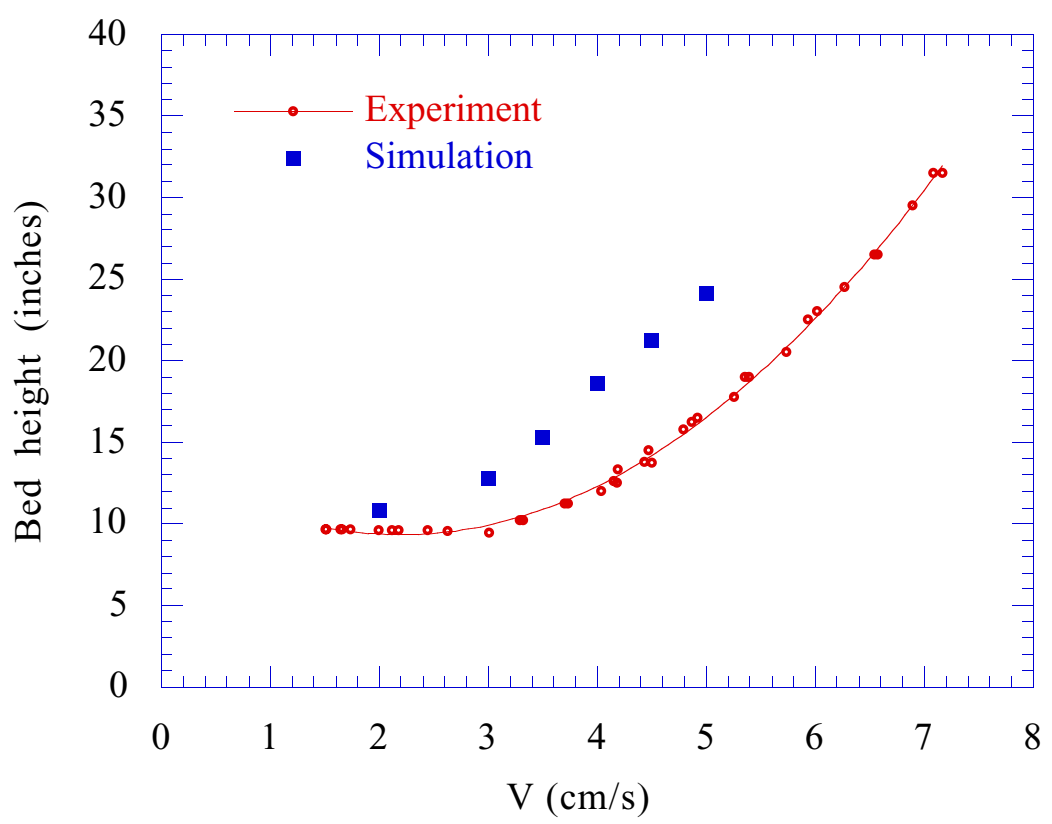

Figure 17. The bed height vs. fluidizing velocity for both experiment and simulation.

The Richardson-Zaki correlation relates the fluidization velocity $V(\phi)$ to the solid fraction $\phi=1-\varepsilon$ where $\varepsilon$ is the fluid fraction, in a factored form in which $V(0)$, the blowout velocity for a single sphere, is multiplied by a hundred settling function. When $V>V(0)$ all the particles will be dragged out of the fluidized bed. For the monodispersed case studied in simulation

$$
H_{s}=4.564 /(1-\varepsilon)
$$

The mean sphere size for the polydisperse case studied in the experiments is slightly larger and

$$
H_{e}=4.636 /(1-\varepsilon)
$$

The Richardson-Zaki correlation is given by

$$
V(\phi)=V(0) \varepsilon^{\mathrm{n}(\mathrm{Re})}
$$

where $V(0)$ is $V$ when $\varepsilon=1$,

$$
\begin{aligned}
& n=\left(4.65+19.5 \frac{d}{D}\right) \text { when } \operatorname{Re}=V(0) d / v<0.2, \\
& n=\left(4.36+17.6 \frac{d}{D}\right) \text { when } \quad 0.2<\operatorname{Re}<1, \\
& n=4.45 \operatorname{Re}^{-1} \text { when } 1<\operatorname{Re}<500, \\
& n=2.39 \text { when } 500<\operatorname{Re}<7000
\end{aligned}
$$

and $D$ is the tube radius. In our experiments and simulations Re is confined to the range for which $n=2.39$. 
The data shown in Figure 17 is plotted in a $\log -\log$ plot in Figure 18 as $V$ vs. $\mathcal{E}$. We draw a straight line with slop $n=2.39$ through both sets of data. The fit is not perfect but we think rather encouraging. From the straight lines we determine the blow-out velocities $V_{s}(0)=8.131 \mathrm{~cm} / \mathrm{s}$ and $V_{e}(0)=10.8 \mathrm{~cm} / \mathrm{s}$ and find the power laws

$$
V_{s}(\phi)=8.131 \varepsilon^{2.39} \mathrm{~cm} / \mathrm{s}
$$

and

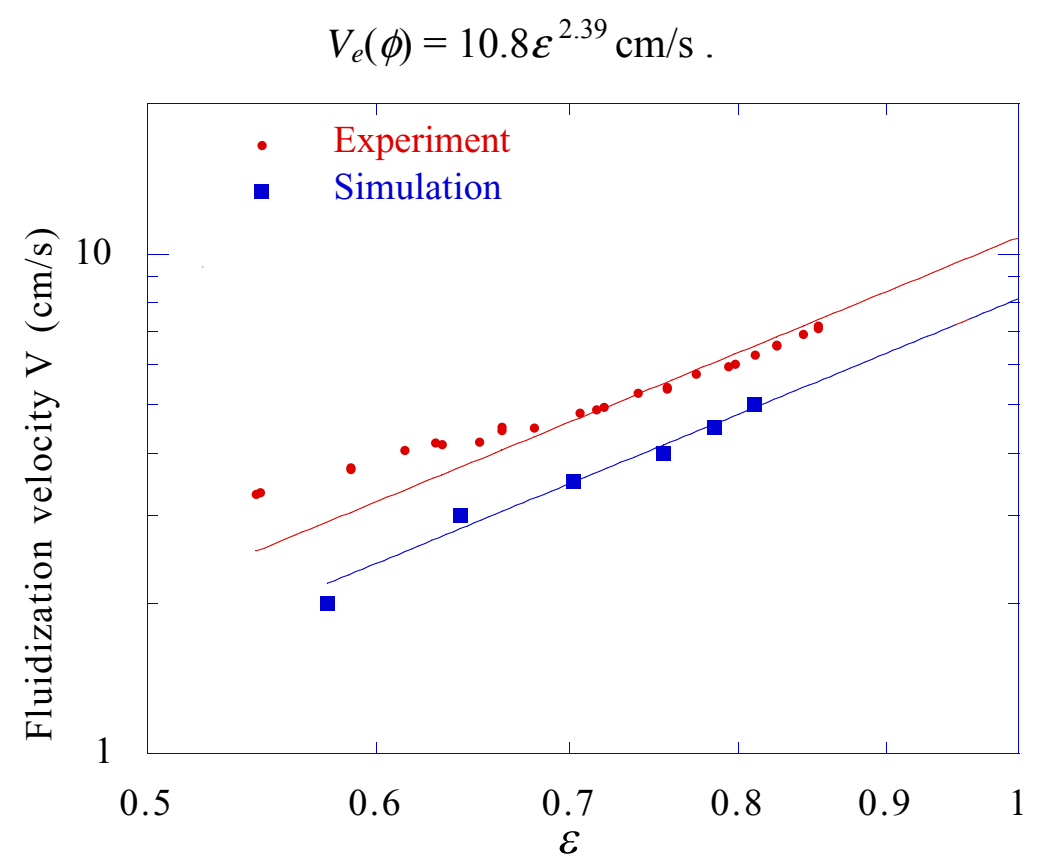

Figure 18. Data from Figure 17 plotted in a log-log plot. The slopes of the straight line are given by the Richardson-Zaki $\mathrm{n}=2.39$. The blow-out velocities $\mathrm{V}_{\mathrm{s}}(0)$ and $\mathrm{V}_{\mathrm{e}}(0)$ are defined as the intercepts at $\varepsilon=1$.

Different reasons could be considered for the discrepancy between numerical and experimental blow out velocities. A referee of this paper thinks that the reason for the discrepancy is that tangential lubrication forces are not accurately calculated by our numerical method. We think that the discrepancy is due to the difference in the diameter $0.635 \mathrm{~cm}$ of the sphere in the simulation and average diameter $0.6398 \mathrm{~cm}$ of the 1204 spheres used in the experiments. This means that the walls will increase the drag more in the experiments than in the simulations. To estimate the effect we may say the wall correction formulas of Francis [1933], which was derived for Stokes settling of a sphere of diameter $d$ in a tube of diameter $D$

$$
V(0)=\frac{\rho_{s}-\rho_{f}}{18 \eta_{f}} d^{2}\left(1-\frac{d}{D}\right)^{2.25}
$$

If we are allowed to consider $D=0.686 \mathrm{~cm}$, which is the distance between plane walls rather than a tube diameter, then the ratio of velocities corresponding to the nominal $d_{1}$ and the average diameter $d_{2}$ is given by 


$$
\frac{V_{s}(0)}{V_{e}(0)}=\left(\frac{d_{1}}{d_{2}}\right)^{2}\left(\frac{D-d_{1}}{D-d_{2}}\right)^{2.25}=\left(\frac{6.35}{6.398}\right)^{2}\left(\frac{51}{46.2}\right)^{2.25}=1.233
$$

The value 1.233 is very close to the shift ratio

$$
\frac{10.8}{8.131}=1.248
$$

necessary to bring the straight lines in Figure 18 together. After shifting by (9.8) we reverse the log-log plot to view the shifted plot in Figure 19.

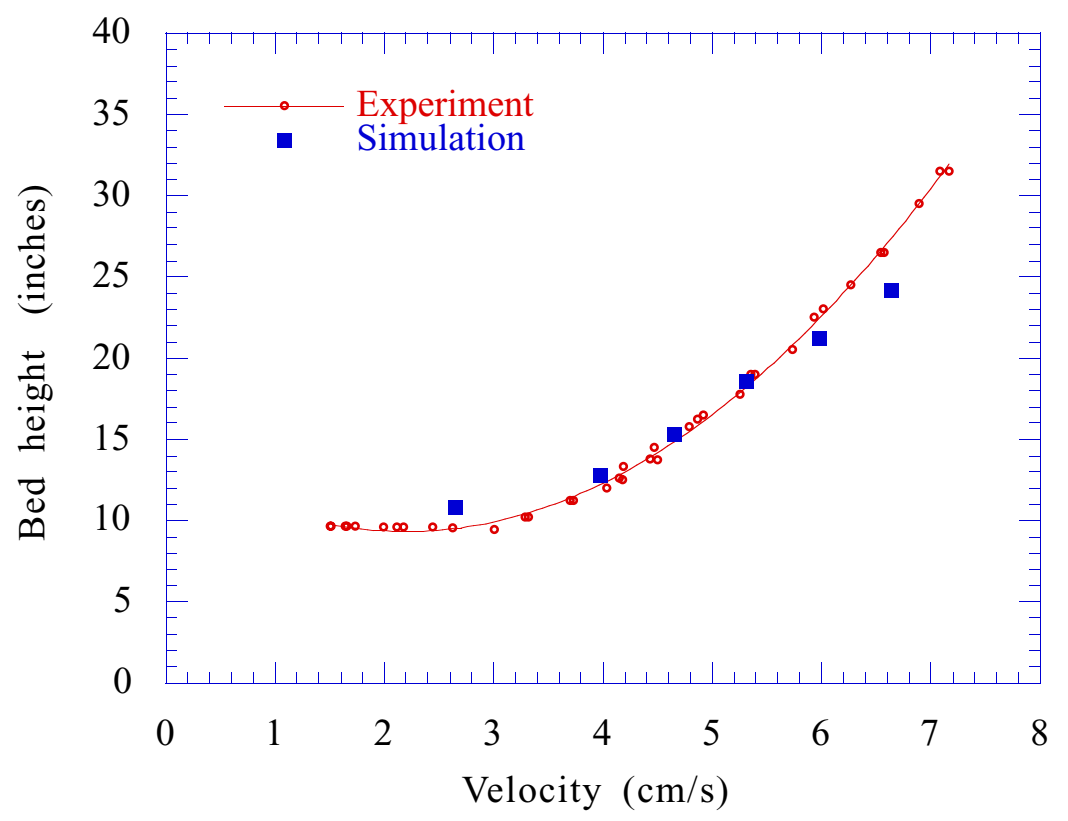

Figure 19. Bed height vs. fluidizing velocity after shifting by the ratio (9.8) of blow-out velocities obtained from the intercepts at $\varepsilon=1$ in Figure 18.

\section{Discussion}

The fluidization of 1204 spheres at Reynolds numbers in the thousands was studied using the method of distributed Lagrange multipliers. The results of the simulation are compared with a real experiment designed to match. This is the first direct numerical simulation of a real fluidized bed at the finite Reynolds number encountered in the applications. It is the first attempt to match a real experiment to a fully resolved simulation. The numerical method used is presently very far in advance of competitors for fully resolved CFD approaches to solid-liquid flow. The experiments are carried out in a slit fluidization column in which the gap between close walls is slightly larger than the fluidized spheres. The match between theory and experiment is very good but not perfect; the spheres in the experiments are polydisperse with an average diameter of $0.6398 \mathrm{~cm}$ whereas all the spheres in the simulation are $0.635 \mathrm{~cm}$. When these differences are factored into the comparison the simulations and experiments are in good quantitative agreement; the qualitative agreements are compelling. The emphasis of this paper is on the interrogation of DNS for results in multiphase fluid mechanics and introduces four new directions. First we have shown how our numerical method can be used to generate averaged 
values of the solid fraction, the average of velocity components of both the solid and fluid and possibly other averaged values used in multiphase models; for example we give the first ever numerical simulation of the slip velocity, which might be used in drift flux models. In a second application we obtain the contribution from wall friction that is usually neglected in onedimensional models of the dynamic pressure in a fluidized flow. We find that in our slit bed the contribution of wall friction is about $1 / 4$ of the total and that the contribution decreases modestly as the bed expands. Our comparative study of sedimentation and fluidization of single spheres revealed an unanticipated result that the balance between drag and buoyant weight can be achieved in an interval of velocities. We framed this result as a positioning property; the particle may be moved up and down the column by changing the fluidization velocity. The fluid mechanics here are not understood; we conjectured that the drag can be maintained as the velocity changes by a simultaneous change in the stand-off distance from the wall. The fourth and most important application was framed as the method of correlations inspired by the way that Richardson and Zaki [1954] processed their experimental results for power law correlations. The idea is to plot results of experiments in log-log plots. Remarkably, straight lines emerge. Apparently the flow of dispersions is governed at the foundation by similarity rules which are not at all evident. We have the idea that we may implement this method using numerical rather than real experiments and we think that our results establish this concept. In fact the results in the experiments and simulations do follow the Richardson-Zaki correlation in a compelling if not perfect match. We prefer to frame our result as a demonstration that we can generate power laws by processing DNS data, rather than confirming correlations already obtained. We intend to promote this approach strongly in the future.

\section{Acknowledgements}

We acknowledge the support by NSF (grants ECS-9527123 and CTS-9873236) and the Minnesota Supercomputing Institute. The work of Glowinski and Pan was also supported by the Texas Board of Higher Education (ARP grant 003652-0383-1999) and NSF DMS-9973318. Joseph's work was also supported by NSF GOALI grant and by DOE, Programming of Engineering Research, Dept. of Basic Energy Sciences. We also thank N.A. Patankar for his helpful discussion.

\section{References}

Articles marked with are also available from the web site, http://www.aem.umn.edu/Solid -Liquid_Flows/references.shtml.

Aidun, C.K., Lu, Y., 1995. Lattice Boltzmann simulation of solid particles suspended in fluid. J. Stat. Phys., 81, 49-61.

Aidun, C.K., Lu, Y., Ding, E., 1998. Direct analysis of particulate suspensions with inertia using the discrete Boltzmann equation. J. Fluid Mech., 373, 287-311.

Barenblatt, G.J. 1996. Scaling, self-similarity, and intermediate asymptotics. Cambridge Texts in Applied Math. Cambridge University Press, UK.

Brady, J.F., 1993. Stokesian Dynamics Simulation of Particulate Flows in Particulate Two-

Phase Flow, (M. Roco ed.) 971-998, Butterworth-Heinemann. 
Bristeau, M.O., Glowinski, R., Periaux, J. 1987. Numerical methods for the Navier-Stokes equations. Applications to the simulation of compressible and incompressible viscous flow, Computer Physics Reports, 6, 73-187.

Chorin, A.J. 1967. A numerical method for solving incompressible viscous flow problems, $J$. Comput. Phys., 2, 12-26.

Chorin, A.J. 1968. On the convergence and approximation of discrete approximation to the Navier-Stokes equations, Math. Comput., 23, 341-353.

Chorin, A.J. 1973. Numerical study of slightly viscous flow, J. Fluid Mech., 57, 785-796.

Crowe, C.T., Chung, J.N. and Troutt, T.R., 1996. Numerical models for two-phase turbulent flows. Annual Review of Fluid Mechanics, 28, 11-43.

Francis, A.W. 1933. Wall effect in falling ball method for viscosity, Physics, 4, 403-406.

Glowinski, R., Hesla, T., Joseph, D.D., Pan, T.-W., Periaux, J. 1997. Distributed Lagrange multiplier methods for particulate flows, in Computational Science for the 21 st Century, M.O. Bristeau, G. Etgen, W. Fitzgibbon, J.L. Lions, J. Periaux, M.F. Wheeler eds., J. Wiley, Chichester, U.K., 270-279.

Glowinski, R., Pan, T.-W., Hesla, T., Joseph, D.D., Periaux, J. 1998. A fictitious domain method with distributed Lagrange multipliers for the numerical simulation of particulate flow, in Domain Decomposition Methods 10, J. Mandel, C. Farhat, X.C. Cai eds., American Mathematical Society, Providence, R.I., 121-137.

Glowinski, R., Pan, T.-W., Hesla, T., Joseph, D.D. 1999. A distributed Lagrange multiplier/fictitious domain method for flows around moving rigid bodies: Application to particulate flows, Int. J. Multiphase Flow, 25, 755-794.

Glowinski, R., Pan, T.-W., Hesla, T., Joseph, D.D., Periaux, J. 2001. A fictitious domain approach to the direct numerical simulation of incompressible viscous flow past moving rigid bodies: application to particulate flow, J. Comp. Phys., 169(2), 363-426.

Hofler, K., Muller, M., Schwarzer, S. and Wachmann, B., 1999. Interacting particle-liquid systems, in High Performance Computing in Science and Engineering '98, E. Krause and W. Jager eds., 54-64, Springer-Verlag, Berlin.

Johnson, A. and Tezduyar, T.E., 1999. Advanced mesh generation and update methods for 3D flow simulations, Computational Mech. 23, 130-143.

Ladd, A.J.C., 1994. Numerical simulations of particulate suspensions via a discretized Boltzmann equation. J. Fluid Mech., 271, 285-309, 311-339.

Ladd, A.J.C., 1996. Hydrodynamic screening in sedimenting suspensions of non-Brownian spheres. Phys. Rev. Lett., 76, 1392-1395.

Ladd, A.J.C., 1997. Sedimentation of homogeneous suspensions of non-Brownian spheres. Phys. Fluids, 9, 491-499.

Lewis, W.K., Gilliand, E.R. and Bauer, W.C., 1949. Indust. Engrg. Chem., 41, 1104.

Marchuk, G.I. 1990. Splitting and alternating direction methods, in Handbook of Numerical Analysis, Vol. I, P.G. Ciarlet and J.L. Lions eds., North-Holland, Amsterdam, 197-462.

Maxey, M.R. and Patel, B.K., 1997. Force-coupled simulations of particle suspensions at zero and finite Reynolds numbers, ASME FEDSM, 97-3188.

Maxey, M.R., Patel, B.K., Chang, E.J. and Wang, L.P. 1997. Simulations of dispersed turbulent multiphase flow, Fluid Dynamics Res., 20, 143-156. 
McLaughlin, J.B., 1994. Inertial computation of particle turbulent interaction, Int. J. Multiphase Flow, 20, 211-232.

Ory, E, Oguz, H.N and Prosperetti, A., 2000. Physalis: a new $O(N)$ method for the numerical simulation of particle flows, Proceedings of ASME FEDSM'00, ASME 2000 Fluids Engineering Divisions Summer Meeting, June 11-15, Boston, MA.

Pan, T.-W., Sarin, V., Glowinski, R., Sameh, A., Periaux, J. 1999. A fictitious domain method with distributed Lagrange multipliers for the numerical simulation of particular flow and its parallel implementation, in Parallel Computational Fluid Dynamics, Development and Applications of Parallel Technology, C.A. Lin, A. Ecer, N. Satofuka, P. Fox, and J. Periaux eds. North-Holland, Amsterdam, 467-474.

Patankar, N.A., Huang, P.Y., Ko, T. and Joseph, D.D., 2001. Lift-off of a single particle in Newtonian and viscoelastic fluids by direct numerical simulation, accepted by J. of Fluid Mech.

Patankar, N., Ko, T., Choi, H.G. and Joseph, D.D., 2001. A correlation for the lift-off of many particles in plane Poiseuille flows of Newtonian fluids, J. Fluid Mech. 438, 101-128.

Peskin, C.S. 1977. Numerical analysis of blood flow in the heart, J. Comput. Phys., 25, 220-252.

Peskin, C.S. 1981. Lectures on mathematical aspects of Physiology, Lectures in Appl. Math., 19, 69-107.

Peskin, C.S., McQueen, D.M. 1980. Modeling prosthetic heart valves for numerical analysis of blood flow in the heart, J. Comput. Phys., 37, 113-132.

Qi, D., 1997. Non-spheric colloidal suspensions in three-dimensional space. Int. J. Mod. Phys. C, 8, 985-997.

Qi, D., 1999. Lattice Boltzmann simulations of particles in non-zero Reynolds number flows. $J$. Fluid Mech., 385, 41-62.

Richardson, J.F. and Zaki, W.N., 1954. Sedimentation and Fluidization: Part I, Trans. Instn. Chem. Engrs. 32, 35-53.

Sarin, V., Sameh, A. 1998. An efficient iterative method for the generalized Stokes problem, SIAM J. Sci. Comput., 19, 206-226.

Singh, P., Hesla, T.I. and Joseph, D.D. 2001. A modified distributed Lagrange multiplier/fictitious domain method for particulate flows with collisions. Submitted to Int. J. Multiphase Flow.

Wachmann, B., Kalthoff, W., Schwarzer, S. and Hermann, H., 1998. Collective drag and sedimentation: comparison of simulation and experiment in two and three dimensions, Granular Matter, 1(2), 75-82.

Wachmann, B. and Schwarzer, S., 1998. Three-dimensional massively parallel computing of suspensions, Int. J. Mod. Phys. C, 9(5), 759-775.

Wilhelm, R.H. and Kwauk, M., 1948. Fluidization of solid particles, Chem. Engrg Progr. 44, 201. 